\title{
Calculation of a Population Externality
}

\author{
By Henning Bohn and Charles Stuart*
}

\begin{abstract}
It is known that when people generate externalities, a birth also generates an externality and efficiency requires a Pigou tax/subsidy on having children. The size of the externality from a birth is important for studying policy. We calculate the size of this "population externality" in a specific case: we consider a maintained hypothesis that greenhouse gas emissions are a serious problem and assume government reacts by optimally restricting emissions. Calculated population externalities are large under many assumptions.
\end{abstract}

JEL: $H 23$

Keywords: population externality, Pigovian policy

Harford $(1997,1998,2000)$ shows that when people generate externalities and parents care about children's utility, a birth induces an externality and efficiency requires a child tax or subsidy equal to the induced externality. This is true even if optimal taxes are imposed on underlying externalities. Harford's result matters for policy if induced population externalities are large. We make a case that population externalities may be large. Specifically, we study a maintained hypothesis that greenhouse gas emissions are a serious enough problem that emissions must be restricted, and we show that the induced population externality is large under many assumptions.

An intuition for Harford's result here is that two decision variables, emissions and fertility, each require a separate instrument if policy is to be efficient. Suppose a positive optimal tax is imposed on emissions and the revenue is redistributed as equal lump-sums. This introduces a fixed-common-property externality: an additional child means more

\footnotetext{
* Bohn: University of California Santa Barbara. Address: Department of Economics, NH2127, UCSB, Santa Barbara, CA 93106, USA. Email: henning.bohn@ucsb.edu. Stuart: University of California Santa Barbara. Address: Department of Economics, NH2127, UCSB, Santa Barbara, CA 93106, USA. Email: stuart@econ.ucsb.edu. We thank two anonymous referees, Javier Birchenall, Ingemar Hansson, Espen Henriksen, Jodi McMillen, and seminar participants at Georgetown University, Lund University, the Norwegian School of Economics and Business Administration (Bergen), and UCSB.
} 
people in the next generation split the same total redistributions so other parents' children have lower utility. Equivalently, an optimal emissions tax restricts emissions, and when emissions are restricted to the optimal level, a birth means more people in the next generation split the same total emissions so living standards are lower. If parents care about children, a birth therefore harms other parents and efficiency requires a positive tax on births. ${ }^{1}$

We use a balanced-growth setting in which output is produced from labor and greenhouse gas emissions. To capture how economic growth contributes to emissions, factor productivities grow exogenously at constant rates as in Solow-type models. ${ }^{2}$ Population and hence labor are determined endogenously by identical dynastic households with Barro-Becker $(1988,1989)$ preferences. Government policy is set by representative living adults who maximize dynastic utility.

We divide time into two stylized eras. Scientific information about the environmental impact of emissions the arrives at the dawn of a period indexed $t=0$. For $t<0$, the possibility of global warming is unrecognized, emissions are unrestricted, and population and emissions grow exponentially. We calibrate the model to initial conditions at $t=0$. For $t \geq 0$, we derive both the population sequence that maximizes the utility of the representative household taking account of the externality (the optimal population) and the population sequence households would choose without population policy (the natural population). ${ }^{3}$ For most of the paper, we simplify by making environmental damage a step function that implies catastrophic damage beyond a critical emissions level. ${ }^{4}$ Optimal

\footnotetext{
${ }^{1}$ Golosov, Jones, and Tertilt (2007) discuss alternative notions of efficiency when fertility is endogenous. Efficiency and optimality in the current paper refer to a state in which living individuals in a period cannot be made better off. This corresponds to Golosov et al's " $\mathcal{A}$-efficiency."

${ }^{2}$ Kelly and Kolstad (2001) argue that the assumptions in integrated assessment models that productivity and population growth fall exogenously to zero are empirically unrealistic and reduce the emissions problem. Consistent with this, they find the welfare cost of a marginal birth is small if there is no cap and productivity and population growth fall exogenously to zero.

${ }^{3}$ Technically, optimal population is derived from a planning problem that can be implemented with emissions taxes (or caps) and child taxes. Natural population solves the same problem subject to a no-child-tax constraint.

${ }^{4}$ The focus on catastrophic impacts follows Nordhaus and Boyer (2000), who summarize evidence on emissions costs and conclude that "market impacts are likely to be relatively small; the major concerns are the potentially catastrophic impacts." Pindyck (2013) and Barro (2013) stress the importance of catastrophic outcomes and note that uncertainty is an analytic obstacle. The maintained hypothesis in the current paper provides an approach for studying catastrophic outcomes without making assumptions about some of the uncertainties. Note that Golosov et al (2011) derive a formula for the marginal externality damage from emissions in a model without fertility choice. Among other things, they find that the optimal carbon tax is higher by a factor of about 10 under Nordhaus' catastrophic scenarios. The current paper
} 
emissions policy is then a fixed cap at the critical level. This allows us to focus on fertility and population policy.

When emissions are restricted, the economy becomes Malthusian in that population growth reduces per-capita emissions and incomes which in turn restrains population growth. Living standards still rise over time, however, because of exogenous productivity growth. Optimal policy leads to a steady state with reduced ratio of emissions to income and with positive child taxes. Convergence takes many generations and optimal child taxes at $t=0$ differ significantly from the steady state, so the dynamics are important. The economy without child taxes also converges to a steady state, but with substantially higher population and lower per-capita income than in the optimal steady state.

We study only one underlying externality. In the real world, people generate many underlying positive and negative externalities, each of which contributes, positively or negatively, to an aggregate induced population externality. Evaluating this aggregate population externality would be a substantial task. The large numerical values of the population externality we find suggest broadly that numerical evaluation of population externalities induced by other underlying externalities may be a valuable tool for studying economic pressures to tax or subsidize children. ${ }^{5}$

Section I describes the setting. Section II briefly describes the balanced-growth path for $t<0$. Section III studies the dynamics of the natural population under an emissions cap and establishes convergence to a new steady state. Section IV examines the optimal population and child taxes. Section $\mathrm{V}$ extends the model by adding time costs of children, a more general technology, and exogenously-given productivity growth, which are important for numerics. Section VI presents results for a base-line calibration and sensitivity analysis. Section VII concludes. All technical claims are proved in an online appendix.

\footnotetext{
differs from Golosov et al in that we add fertility as a choice variable to study implications for population policy.

${ }^{5}$ Two underlying externalities that may contribute positively and substantially to the aggregate population externality are scale or spillover effects in human capital formation (e.g. Kremer, 1993; Jones, 1999), and the pay-as-you-go externality under which the lifetime social security taxes paid by an additional person benefit all elderly in the previous generation instead of just the parent (Schoonbroodt and Tertilt, 2011).
} 


\section{Setting}

A large number $N_{t}$ of identical (representative) households in period $t$ each contain one adult who supplies labor, consumes, and has children. Aggregate labor is $L_{t}=l_{t} N_{t}$ where $l_{t}$ is per-capita labor. (Per-capita means per-adult.) Firms produce output $Y_{t}$ under perfect competition from $L_{t}$ and from $E_{t}$ units of greenhouse gas emissions according to $Y_{t}=F\left(L_{t}, E_{t}\right)\left(1-\delta_{t}\right)$, where $F$ captures the productivities of labor and emissions as inputs and $\delta_{t}$ is the share of output lost (damage) from global warming. Government sets policy to maximize the utility of the adults living in a period. In more detail:

\section{A. Production}

Production exhibits constant returns so $F\left(L_{t}, E_{t}\right)=L_{t} f\left(e_{t}\right)$, where $e_{t} \equiv E_{t} / L_{t}$ is the emissions ratio and $f$ is output per unit of labor. We assume $f(0)=0$ for now but relax this in section V. We also add exogenously-growing factor productivity in section V.

The marginal product of emissions must be driven to zero if emissions are unrestricted so we assume there is a positive value $e^{+}<\infty$ at which $f^{\prime}\left(e^{+}\right)=0$ with $f^{\prime}\left(e_{t}\right)>0$ and $f^{\prime \prime}\left(e_{t}\right)<0$ for $0 \leq e_{t}<e^{+}$, as in figure 1. If emissions are restricted, output is reduced as indicated by the arrows in figure 1 .

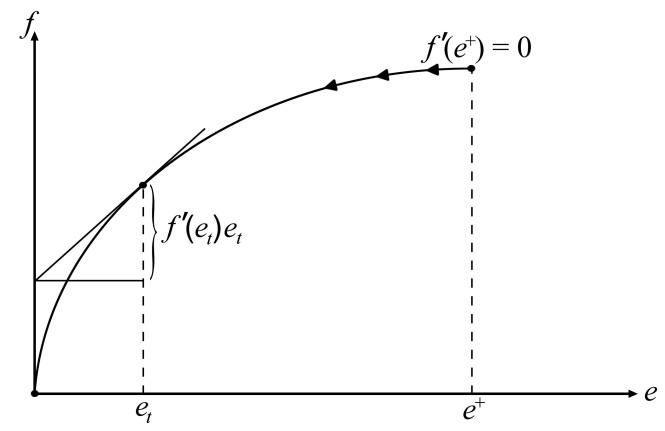

Figure 1. Input Distortion, and Geometry of the Real Population Externality $f^{\prime}(e) e$.

\section{B. Environmental Damages and Dynamics}

A general representation is that damages $\delta_{t}=\delta\left(\mathbf{X}_{t}\right)$ are a function of environmental state variables $\mathbf{X}_{t}$ that follow a process $\mathbf{X}_{t}=\Theta\left(\mathbf{X}_{t-1}, E_{t-1}\right)$. Science suggests that $\mathbf{X}_{t}$ is 
a vector of high dimension and that $\Theta$ includes multiple feedback loops and complicated non-linear relationships.

We first formulate the government's policy problem based on this general representation, then simplify to focus on population. We initially impose three simplifying assumptions:

S1: Damages are catastrophic for a set of environmental states $\mathcal{X}: \delta(\mathbf{X})=1$ for $\mathbf{X} \in \mathcal{X}$.

S2: There is a critical emissions level $\hat{E}$ such that $\Theta\left(\mathbf{X}_{t}, E_{t}\right) \in \mathcal{X}$ if and only if $E_{t}>\hat{E}$.

S3: Non-catastrophic damages are zero: $\delta(\mathbf{X})=0$ for $\mathbf{X} \notin \mathcal{X}$.

$$
\text { Under (S1-S3), damages } \delta_{t+1}=\left\{\begin{array}{ll}
0 & \text { if } E_{t} \leq \hat{E} \\
1 & \text { if } E_{t}>\hat{E}
\end{array}\right. \text { are a step function of emissions, }
$$

which makes optimal emissions policy simple (a cap at $\hat{E}$, as we verify below).

Assumptions S1-S2 formalize the maintained assumption that global warming is serious enough that emissions must be restricted. S1 captures the idea that catastrophic damages are a threat. S2 links catastrophe in the next generation to emissions, regardless of initial conditions. This is a strong assumption designed to simplify emissions policy. $\mathrm{S} 3$ avoids the distraction of small damages. We relax (S1-S3) in Section VI and compute optimal policies numerically for more general specifications for $\delta$ and $\Theta$. The existence of $\mathcal{X}$ and the value $\hat{E}$ are discovered at $t=0$. Choices for $t<0$ are made under the belief that $\delta=0$ for all $(\mathbf{X}, E)$.

\section{The Mechanism of Emissions Policy}

To model emissions policy, we consider a simple auction procedure. Emissions without a permit are forbidden. The government auctions $E_{t}^{p}$ permits in period $t$, each permit allowing one unit of emissions in the period, and distributes the revenue as equal lump sums to households. ${ }^{6}$

\footnotetext{
${ }^{6}$ This treats permits as valid for a single period, which fits U.S. legislative proposals that state permits are not property rights and nothing restricts future government from terminating or limiting an emission allowance.

We show in the appendix that the population externality would be internalized by parents if current government can and does establish iron-clad permanent private property rights to the public revenue stream from all permits to $t=\infty$. This is an interesting idea but is difficult to achieve in practice. Permanence fails if government later changes policy to expropriate "permanent" rights. The U.S. legislative proposals effectively acknowledge that this cannot be prevented.
} 
A representative firm maximizes profits $f\left(e_{t}\right)\left(1-\delta_{t}\right) L_{t}-p_{t} E_{t}-w_{t} L_{t}$, where $p_{t}$ is the price of a permit and $w_{t}$ is the wage. The first-order conditions are $p_{t}=f^{\prime}\left(e_{t}\right)\left(1-\delta_{t}\right)$ and $w_{t}=\left[f\left(e_{t}\right)-e f^{\prime}\left(e_{t}\right)\right]\left(1-\delta_{t}\right)$. The former defines the demand for emission permits. If $E_{t}^{p} \geq e^{+} L_{t}$, emission restrictions do not bind: $p_{t}=0$ and $e_{t}=e^{+}$, so firms emit $E_{t}=e^{+} L_{t}$ and pay a wage $w_{t}=f\left(e^{+}\right)\left(1-\delta_{t}\right)$. If $E_{t}^{p}<e^{+} L_{t}$, emission restrictions bind: $E_{t}=E_{t}^{p}, e_{t}=E_{t}^{p} / L_{t}<e^{+}, p_{t}>0$, and $w_{t}<f\left(e^{+}\right)\left(1-\delta_{t}\right)$. By choosing $E_{t}^{p}$, government effectively sets emissions to $E_{t}=\min \left\{E_{t}^{p}, e^{+} L_{t}\right\}$. Thus the auction quantity $E_{t}^{p}$ can be interpreted as a cap on emissions.

Note that the government could use taxes to set emissions instead of auctioning permits: imposing a tax of $p_{t}=f^{\prime}\left(\min \left\{e^{+}, E_{t}^{p} / L_{t}\right\}\right)\left(1-\delta_{t}\right)$ per unit emissions would also hold emissions to $E_{t}=\min \left\{E_{t}^{p}, e^{+} L_{t}\right\}$. Tax and auction mechanisms are thus equivalent; either way, the government can choose $E_{t} \in\left[0, e^{+} L_{t}\right]$ and obtains revenue $p_{t} E_{t}$.

Government revenue $p_{t} E_{t}$ implies per-capita transfers of $p_{t} E_{t} / N_{t}=p_{t} e_{t}$. Hence wages plus transfers equal output per-capita: $y_{t}=w_{t} l_{t}+p_{t} e_{t}=f\left(e_{t}\right)\left(1-\delta_{t}\right)=Y_{t} / N_{t}$.

\section{Preferences}

A large number of representative dynastic households trade off own consumption and number of children. Own consumption $c_{t} \geq 0$ is per-capita income $y_{t}$ less the output cost of having and raising $n_{t} \geq 0$ children to adulthood: $c_{t}=y_{t}-\chi n_{t}$, where $\chi$ is the output cost of a child and $n_{t}$ is a continuous choice variable. Maximum feasible fertility is $y_{t} / \chi$.

We assume initially that per-capita labor does not depend on fertility and is normalized to one $\left(l_{t}=1\right)$, so aggregate labor equals population $\left(L_{t}=N_{t}\right)$. An extension in Section $\mathrm{V}$ adds time costs of children which reduce $l_{t}$.

We follow Barro and Becker's $(1988,1989)$ specification of household preferences. A period- $t$ adult's utility $U_{t}$ is utility $u$ from own consumption plus utility from children:

$$
U_{t}=u\left(c_{t}\right)+\beta\left(n_{t}\right) U_{t+1}
$$


Utility from children is the utility of a child $\left(U_{t+1}\right)$ times a weight $\beta$ that depends on the number of children. Adults die at the end of a period; their children become economically active as adults in the next period. When all households choose the same $n_{t}$, the population growth factor is also $n_{t}$, that is, $N_{t+1}=n_{t} N_{t}$.

We assume power utility with curvature parameter $\theta>0$ :

$$
u(c)=\frac{1}{1-\theta} c^{1-\theta}
$$

the power form is needed later to allow for balanced growth. We also assume $\beta$ is a power function with curvature parameter $b>0:^{7}$

$$
\beta(n)=b_{0} n^{1-b},
$$

where $0<b_{0}<1$. In Barro and Becker's original specification, $u$ is positive so $\theta<1$, and $\beta$ is increasing and concave so $b<1$. These assumptions ensure that parent's utility rises at a decreasing rate with the number of children. Jones, Schoenbroodt, and Tertilt (2008) and Jones and Schoonbroodt (2010) show that parent's utility also rises at a decreasing rate with the number of children if utility is negative so $\theta>1$ and if $\beta$ is decreasing and convex so $b>1$. We allow either $(\theta<1, b<1)$ or $(\theta>1, b>1){ }^{8}$

From (1) - (3), contribution of children to parent's utility can be written $\beta\left(n_{t}\right) u\left(c_{t+1}\right)=$ $\left(n_{t} c_{t+1}^{\omega}\right)^{1-b} b_{0} /(\omega(1-b))$ where $\omega \equiv(1-\theta) /(1-b)$ is the weight a parent places on per-child consumption relative to the number of children. In the case of equal curvatures $(\theta=b$, so $\omega=1)$ the contribution depends on children's aggregate consumption, $n_{t} c_{t+1}$. This case may be reasonable if parents view children's aggregate consumption as an economic resource; it will serve as baseline for our numerical analysis. ${ }^{9}$

\footnotetext{
${ }^{7}$ Power $\beta$ has a sensible property: it is equivalent to assuming the utility an adult derives from grandchildren, $\beta\left(n_{t}\right) \beta\left(n_{t+1}\right) U_{t+2}$, is independent of the number of children-see appendix.

${ }^{8}$ For $(\theta<1, b<1)$, utility (1) is infinite unless $\beta(n)<1$ for all feasible $n$; to ensure bounded $U$, we assume $\chi>b_{0}^{1 /(1-b)} f\left(e^{+}\right)$for $b<1$, which implies $\beta\left(f\left(e^{+}\right) / \chi\right)<1$. For $(\theta>1, b>1), U$ is always bounded above.

${ }^{9}$ We do not specifically rule out cases with $\omega$ greater or less than one. Values of $\omega$ far less than one imply that parents care little about their children's consumption relative to the number of children. However, this may be difficult to square with small families in which parents devote substantial resources to ensuring children's consumptions. Values $\omega>1$
} 


\section{E. Government Policy}

The government maximizes the utility of current adults. We consider two settings for policy. One is the government's (basic) planning problem, which is to maximize utility subject to a resource constraint. The solution addresses all externalities optimally. Its implementation as market equilibrium generally requires child taxes or equivalent tools of population policy, in addition to emissions policy. Controls on emissions are empirically plausible and naturally motivated by environmental externalities. In contrast, child taxes are not a generally-accepted policy tool, and controls on fertility such as the one-child policy in China have a history of controversy. An allocation with optimal emissions policy but no child taxes is therefore a natural benchmark for studying optimal population. It is derived below as solution to a planning problem with no-child-tax constraint.

Consider first the basic planning problem. The government in a period $t_{0}$ maximizes $U_{t_{0}}$ for given $\left(N_{t_{0}}, \mathbf{X}_{t_{0}}\right)$ by choice of sequences $\left\{N_{t+1}, E_{t}\right\}_{t \geq t_{0}}$ subject to $c_{t}=$ $f\left(E_{t} / N_{t}\right)\left(1-\delta\left(\mathbf{X}_{t}\right)\right)-\chi N_{t+1} / N_{t}$. The problem is recursive but non-standard because of the endogenous discount factor $\beta\left(n_{t}\right)$ in (1). Alvarez (1999) has shown that solutions (if any) can be obtained by solving the transformed problem of maximizing $V_{t} \equiv \beta\left(N_{t}\right) \cdot U_{t}$. For general damages $\delta$ and dynamics $\Theta$, this problem has the Bellman equation:

$$
V\left(N_{t}, \mathbf{X}_{t}\right)=\max _{N_{t+1}, E_{t}}\left\{\beta\left(N_{t}\right) u\left(c_{t}\right)+b_{0} V\left(N_{t+1}, \Theta\left(E_{t}, \mathbf{X}_{t}\right)\right)\right\}
$$

where $E_{t} \in\left[0, e^{+} N_{t}\right]$ and $N_{t+1} \in\left[0, f\left(E_{t} / N_{t}\right)\left(1-\delta\left(\mathbf{X}_{t}\right)\right) N_{t} / \chi\right]$.

Problem (4) provides a conceptual framework, but assumptions about $(\delta, \Theta)$ are needed to obtain insightful results. In particular, we impose S1-S3 (until Section VI-E).

Under S1-S3 and assuming $\mathbf{X}_{t} \notin \mathcal{X}$ (no catastrophe), $c_{t}=f\left(E_{t} / N_{t}\right)-\chi N_{t+1} / N_{t}$ does not depend on $\mathbf{X}_{t}$ so $V$ depends only on population. Optimal emissions policy is then a simple cap, $E_{t}^{*}=\min \left\{e^{+} N_{t}, \hat{E}\right\}$, which maximizes current output while avoiding

imply that the marginal utility to parents of children's consumption is increasing. As described below, this may cause non-concavity of the optimal population problem. 
catastrophe. Given the optimal cap, the planning problem can be written concisely as

$$
V^{*}\left(N_{t}\right)=\max _{N_{t+1}}\left\{\beta\left(N_{t}\right) u\left(f\left(\min \left\{e^{+}, \frac{\hat{E}}{N_{t}}\right\}\right)-\chi \frac{N_{t+1}}{N_{t}}\right)+b_{0} V^{*}\left(N_{t+1}\right)\right\} .
$$

The solution to (5) defines the optimal population $\left\{N_{t}^{*}\right\}_{t>t_{0}}$. Throughout, superscripts * and the label optimal refer to solutions to the basic planning problem, or equivalently, to allocations with jointly optimal emissions and child taxes. (For completeness, we must verify uniqueness and that catastrophe is undesirable-see appendix.)

Now consider the planning problem without child taxes. Policy is then constrained by household fertility choices. Generically (dropping time subscripts), a household chooses $n$ to maximize

$$
U^{\circ}(n, y, U) \equiv u(y-\chi n)+\beta(n) U
$$

taking income $y$ and the utility of future generations $U$ as given. The first-order condition

$$
U_{n}^{\circ}(n, y, U) \equiv \frac{d U^{\circ}}{d n}=-u^{\prime}(y-\chi n) \chi+\beta^{\prime}(n) U=0 .
$$

defines a unique individually-optimal fertility $n^{\circ} \in(0, y / \chi)$ for given $(y, U)$. In the planning problem, zero child taxes imply that (7) is an implementation constraint.

The government's problem without child taxes and with general $(\delta, \Theta)$ is then to maximize (4) subject to (7), where (7) is evaluated at $n=N_{t+1} / N_{t}, y=\left(1-\delta\left(\mathbf{X}_{t}\right)\right) f\left(E_{t} / N_{t}\right)$, and $U=V^{\circ}\left(N_{t+1}, \Theta\left(E_{t}, \mathbf{X}_{t}\right)\right)$, and $V^{\circ}$ denotes the value function. Under S1-S3, again $\mathbf{X}_{t}$ drops out, so optimal emissions are $E_{t}^{\circ}=\min \left\{e^{+} N_{t}, \hat{E}\right\}$ by the reasoning above. The natural population $\left\{N_{t}^{\circ}\right\}_{t>t_{0}}$ is then determined iteratively by the individual choices in (7). Throughout, superscripts ${ }^{\circ}$ and the label natural refer to allocations without child taxes. Note that under S1-S3, optimal and natural populations are directly comparable because emissions policies are the same. 


\section{Population In the No-cap Era}

The no-cap era means periods $t<0$ when damages from emissions are not recognized. Then the recursive utility (1) and the first-order condition (7) imply a perfectforesight solution with constant utility $U^{+}$and fertility $n^{+}$. Specifically, constant utility in (1) implies $U=u(f(e)-\chi n) /(1-\beta(n))$. Substituting this into (7) yields

$$
S(n, e) \equiv-u^{\prime}(f(e)-\chi n) \chi+\frac{\beta^{\prime}(n)}{1-\beta(n)} u(f(e)-\chi n)=0 .
$$

Fertility $n^{+}$solves $S\left(n^{+}, e^{+}\right)=0$; such an $n^{+}$exists and is unique. Then $U^{+}=$ $u\left(f\left(e^{+}\right)-\chi n^{+}\right) /\left(1-\beta\left(n^{+}\right)\right) .{ }^{10}$

In general, $n^{+}$may be greater or less than one. Fertility is greater than one as long as child costs are not too great a fraction of output. To focus on equilibria in which population and emissions grow so emissions can be a problem, we assume

$$
\chi<\phi f\left(e^{+}\right) \text {where } \phi \equiv 1 /\left(1+\frac{(1-\theta)\left(1-b_{0}\right)}{(1-b) b_{0}}\right)<1
$$

Equation (9) rearranges to $S\left(1, e^{+}\right)>0$, which ensures $n^{+}>1$. Population and emissions then grow without bound at constant rate $n^{+}-1>0$.

\section{Natural Population in the Cap Era}

The cap era starts in period $t=0$, when damages from greenhouse gas emissions are discovered. Under assumptions (S1-S3) and no child taxes, government optimally caps emissions at $\hat{E}$.

Generally, population cannot grow without bound when emissions are bounded, because unbounded population would drive output per unit of labor $f(e)$ below $\chi$ and so fertility would fall below replacement. Under a regularity condition described below, natural population instead converges monotonically to a unique steady-state level $N_{s s}^{\circ}$.

\footnotetext{
${ }^{10}$ Under zero damages, $N_{t+1}=n^{+} N_{t}, E_{t}=e^{+} N_{t}$, and $V\left(N_{t}, X_{t}\right)=\beta\left(N_{t}\right) U^{+}$solve (4), so the individuallyrational fertility is also optimal.
} 
(Subscripts $s s$ denote a variable's steady-state value.)

The household problem in the cap era satisfies the recursion (1) and the first-order condition (7) for all $t$, which can be written as pair of first-order difference equations in $\left\{U_{t}, N_{t}\right\}_{t \geq 0}$ :

$$
\begin{aligned}
U_{t} & \left.=u\left(f\left(e\left(N_{t}\right)\right)-\chi \frac{N_{t+1}}{N_{t}}\right)+\beta\left(\frac{N_{t+1}}{N_{t}}\right)\right) U_{t+1} \\
U_{n}^{\circ}(t) & \equiv-u^{\prime}\left(f\left(e\left(N_{t}\right)\right)-\chi \frac{N_{t+1}}{N_{t}}\right) \chi+\beta^{\prime}\left(\frac{N_{t+1}}{N_{t}}\right) U_{t+1}=0 .
\end{aligned}
$$

where $e\left(N_{t}\right) \equiv \min \left\{e^{+}, \hat{E} / N_{t}\right\}{ }^{11}$

A steady state is a pair $\left(U_{s s}^{\circ}, N_{s s}^{\circ}\right)$ that satisfies (10) and (11) with $U_{t}=U_{t+1}=U_{s s}^{\circ}$ and $N_{t}=N_{t+1}=N_{s s}^{\circ}$. The latter implies that steady-state fertility equals replacement: $n_{s s}^{\circ}=1$. Moreover, $e_{s s}^{\circ}=e\left(N_{s s}^{\circ}\right)$ is a constant, $U_{s s}^{\circ}=u\left(f\left(e_{s s}^{\circ}\right)-\chi\right) /(1-\beta(1))$, and $e_{s s}^{\circ}$ must satisfy the steady-state condition $S^{\circ}\left(e_{s s}^{\circ}\right) \equiv S\left(1, e_{s s}^{\circ}\right)=0$. We show in the appendix that $S^{\circ}$ crosses zero exactly once on $\left[f^{-1}(\chi), e^{+}\right]$so $e_{s s}^{\circ}$ exists and is unique. Thus $N_{s s}^{\circ}=\hat{E} / e_{s s}^{\circ}$ exists and is unique. Because $S\left(1, e^{+}\right)>0$, it must be that $e_{s s}^{\circ}<e^{+}$ so the cap binds in steady state.

A perfect-foresight path is a sequence $\left\{U_{t}^{\circ}, N_{t}^{\circ}\right\}_{t \geq 0}$ that satisfies (10) and (11) for all $t$. Population growth in (11) depends on individual fertility, which is driven by income and children's utility. The impact of income on fertility is given by the partial elasticity

$$
\varepsilon_{n, y} \equiv \frac{y}{n} \frac{\partial n}{\partial y}=-\frac{y U_{n y}^{\circ}}{n U_{n n}^{\circ}}=\left[\frac{b}{\theta} \cdot \frac{c}{y}+\frac{\chi n}{y}\right]^{-1}
$$

in (7), which is positive by assumptions on primitives. (This elasticity differs conceptually from many empirically estimated income elasticities: $\varepsilon_{n, y}$ holds children's utility constant, netting out how fertility changes with income via changes in children's wellbeing.)

The impact of children's utility on fertility depends on $b$. This is because the marginal

\footnotetext{
${ }^{11}$ Since optimal emissions policy under S1-S3 is a cap at $\hat{E}$, solving the household problem suffices to derive the equilibrium allocation; writing this as planning problem would needlessly complicate the exposition.
} 
benefit of a child in (7) and (11) is $\beta^{\prime} U$, which rises with $U$ when $b<1$ (so $\beta^{\prime}>0$ ) and falls with $U$ when $b>1$ (so $\beta^{\prime}<0$ ). ${ }^{12}$ Ceteris paribus, a reduction in children's utility reduces fertility if $b<1$ but raises fertility if $b>1$.

If fertility is too sensitive to changes in population then a population increase from $t$ to $t+1$ could reduce fertility so much that population decreases from $t+1$ to $t+2$, which means that population would "overshoot" its steady-state value. To rule out overshooting, we restrict the sensitivity of fertility to changes in population by assuming

$$
\varepsilon_{n_{t}, y_{t}}\left(\frac{f^{\prime}\left(e_{t}\right) e_{t}}{f\left(e_{t}\right)}\right)<1
$$

at the steady state and at all $t .{ }^{13}$ We show in the appendix that the system (10) and (11) is then saddle-path stable and that population converges monotonically to $N_{s s}^{\circ}$ from any initial population $N_{0}>0$.

Fertility along the perfect-foresight path is a function of population, $n_{t}^{\circ}=\eta^{\circ}\left(N_{t}\right)$. Population dynamics follow from $\eta^{\circ}$ as shown in figure 2. Suppose the need to cap emissions at $\hat{E}$ is discovered when population has grown to $N_{0}=\hat{E} / e^{+}$, which means that uncapped emissions would equal $\hat{E}$. To minimize clutter, choose units so $e^{+}=1$, $\hat{E}=1$, and $N_{0}=1$. Without a cap, fertility would be $n^{+}$in period 0 , at point $a$. When the cap is imposed, fertility instead jumps to $\eta^{\circ}\left(N_{0}\right)=\eta^{\circ}(1)$, at $b$. In period 1 , the economy is therefore at $c$ with population $N_{1}=\eta^{\circ}\left(N_{0}\right) \cdot N_{0}$ and fertility $\eta^{\circ}\left(N_{1}\right)$, at $c$. The economy then iterates down the fertility function and converges to population $N_{s s}^{\circ}$ with fertility $\eta^{\circ}\left(N_{s s}^{\circ}\right)=1$. If the need to cap emissions were discovered earlier (when $\left.N_{0}<1\right)$ or later $\left(N_{0}>1\right)$, the jump in fertility from $n^{+}$to $\eta^{\circ}$ would take place at a lower or higher population, and then the economy would iterate along $\eta^{\circ}$ to the same steady state.

\footnotetext{
${ }^{12}$ Formally, (7) implies $\frac{\partial n}{\partial U}=\beta^{\prime}(n) /\left(-U_{n n}^{\circ}\right)$, where $U_{n n}^{\circ}<0$.

${ }^{13}$ In (13), $\varepsilon_{n_{t}, y_{t}}$ is the partial elasticity of fertility with respect to income (12) and the factor share $f^{\prime}\left(e_{t}\right) e_{t} / f\left(e_{t}\right)$ is also the elasticity of income with respect to population. Note that (13) is not very strong. Because $f^{\prime}(e) e<f(e),(13)$ holds if $\varepsilon_{n, y} \leq 1$, which holds in turn if $b \geq \theta$. On the other hand, if $\varepsilon_{n, y}>1$ then fertility tends to fall off sharply as a declining emissions ratio reduces income so the factor share remains small, and (13) can still easily hold.
} 


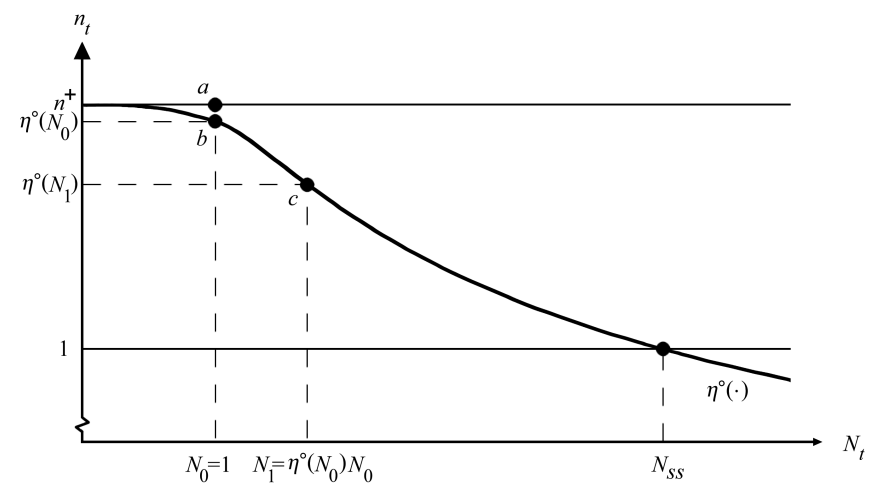

Figure 2. Population Dynamics Under an Emissions Freeze

The shape of the fertility function $\eta^{\circ}$ depends on $b$. There are two forces, operating through (current) income and children's utility. First, a binding cap $\left(\hat{E}<e^{+} N_{t}\right)$ reduces income. This acts to reduce fertility increasingly as $N_{t}$ rises above $\hat{E} / e^{+}$. Second, households know the cap will eventually bind. Because utility is determined recursively, children's utility is therefore less than $U^{+}$as soon as the need to cap emissions is discovered (even if $e^{+} N_{t}<\hat{E}$ ). When $b<1$, households react to the utility decline by reducing fertility, so fertility lies below $n^{+}$and falls with $N_{t}$ to the steady state at $N_{s s}^{\circ}$, as in figure 2. When $b>1$, reduced utility raises fertility, so $\eta^{\circ}$ is above $n^{+}$for $N \leq \hat{E} / e^{+}$and in a neighborhood of $\hat{E} / e^{+}$, but the negative income effect dominates at high $N$-values and ensures that $N_{s s}^{\circ}$ exists. ${ }^{14}$

As noted, this section focuses on policy under $(\mathrm{S} 1-\mathrm{S} 3)$. For general $(\Theta, \delta)$, conditions for convergence would depend on environmental dynamics. Note, however, that if optimal policy leads to a steady state $\left(E_{s s}^{\circ}, \mathbf{X}_{s s}^{\circ}\right)$ for the physical environment, (1) and (7) would imply a steady-state condition for $e_{s s}^{\circ}$ similar to $S^{\circ}=0$ (see appendix). Population would have a finite steady state $N_{s s}^{\circ}=E_{s s}^{\circ} / e_{s s}^{\circ}$, and fertility would be influenced by the same forces as in this section.

\footnotetext{
${ }^{14}$ A numerical illustration of $\eta^{\circ}$ with $b>1$ is in figure 5 below. The utility effect explains why $N_{s S}^{\circ}$ tends to be high in the numerical analysis when $b>1$.
} 


\section{Optimal Population in the Cap Era}

Now consider optimal policy under assumptions (S1-S3). While solutions to the Bellman equation (5) exist for all $(\theta, b)$, their description requires technical restrictions. For $\omega=(1-\theta) /(1-b) \leq 1$, the value function $V^{*}$ turns out to be unique, strictly concave, and differentiable, and optimal population is a single-valued continuous function $N_{t+1}^{*} \equiv H\left(N_{t}\right)$. Moreover, there is a unique steady-state $N_{s s}^{*}$, and the elasticity condition (13) is sufficient for population to converge monotonically to $N_{s s}^{*}$ from any initial $N_{0}>0$. For $\omega>1$, matters are more complicated, but similar results hold provided $N_{0}$ is suitably bounded away from zero and $\omega$ is not too far above one. ${ }^{15}$ The following assumes differentiable $V^{*}$ and single-valued optimal policy $H(N)$.

From the first-order and envelope conditions implied by (5), one can show that steadystate population $N_{s s}^{*}$ must satisfy $S^{*}\left(\hat{E} / N_{s s}^{*}\right)=0$, where

$$
\begin{aligned}
S^{*}(e) & \equiv-\left(1-b_{0}\right) \chi u^{\prime}(c)+b_{0} u(c)-b_{0} u^{\prime}(c) f^{\prime}(e) e \\
& =\left(1-b_{0}\right) S^{\circ}(e)-b_{0} u^{\prime}(f(e)-\chi) f^{\prime}(e) e .
\end{aligned}
$$

This optimal steady-state condition differs from the natural steady-state condition $\left(S^{\circ}=\right.$ $0)$ by a term that reflects the population externality. A root $e_{s s}^{*}=\hat{E} / N_{s s}^{*}$ that solves $S^{*}\left(e_{s s}^{*}\right)=0$ exists and lies strictly between $e_{s s}^{\circ}$ and $e^{+} .{ }^{16}$ Because $e_{s s}^{\circ}<e_{s s}^{*}$, steady-state optimal population $N_{s s}^{*}$ is less than steady-state natural population $N_{s s}^{\circ}$.

The optimal population sequence $\left\{N_{t}^{*}\right\}_{t \geq 0}$ starting from given $N_{0}>0$ can be computed by iterating on $H$. Optimal fertility $n_{t}^{*}=\eta^{*}\left(N_{t}\right) \equiv H\left(N_{t}\right) / N_{t}$ follows from the optimal population. As with natural fertility, optimal fertility: (i) jumps at $t=0$ from $n^{+}$to a new value, $\eta^{*}\left(N_{0}\right)$; and (ii) approaches $n_{s s}^{*}=1$ as population converges to $N_{s s}^{*}$.

As in the previous section, we focus on (S1-S3); again, if the general problem (4)

\footnotetext{
${ }^{15}$ Complications arise because the return function $v\left(N_{t}, N_{t+1}\right) \equiv \beta\left(N_{t}\right) u\left(f\left(\min \left\{e^{+}, \hat{E} / N_{t}\right\}\right)-\chi N_{t+1} / N_{t}\right)$ in (5) is not strictly concave unless $\omega<1$. If $\omega=1, V^{*}$ is nonetheless strictly concave, but if $\omega>1, V^{*}$ is not concave for small $N$. Applications of the Barro-Becker model commonly assume $\omega \leq 1$ (e.g. Jones and Schoonbroodt, 2010). We discuss $\omega>1$ to emphasize that cases with $\theta=b$ in Section VI are not borderline cases.

${ }^{16}$ The details are that $S^{*}$ is continuous, $S^{*}\left(e_{s s}\right)=-\beta u^{\prime} f^{\prime} e<0$ (because $S\left(1, e_{s S}\right)=0$ and $e_{S S}<e^{+}$), and $S^{*}\left(e^{+}\right)>0$ (because $S\left(1, e^{+}\right)>0$ and $f^{\prime}\left(e^{+}\right)=0$ ).
} 
leads to a steady state $\left(E_{s s}^{*}, \mathbf{X}_{s s}^{*}\right)$, steady-state values $e_{s s}^{*}$ and $N_{s s}^{*}=E_{s s}^{*} / e_{s s}^{*}$ would be determined by a condition similar to (14).

\section{A. The Population Externality: Comparing Natural and Optimal Populations}

We evaluate the population externality by examining individual utility $U^{*}\left(N_{t}\right)$ along the optimal path. From (5), $U^{*}=V^{*} / \beta$ must satisfy the recursion

$$
U^{*}\left(N_{t}\right)=\max _{n_{t}}\left\{u\left(c_{t}\right)+\beta\left(n_{t}\right) U^{*}\left(n_{t} N_{t}\right)\right\}
$$

Hence optimal fertility must satisfy the first-order condition

$$
\begin{aligned}
U_{n}^{*} & \equiv-u^{\prime}\left(c_{t}^{*}\right) \chi+\beta^{\prime}\left(n_{t}^{*}\right) U^{*}\left(N_{t+1}^{*}\right)+\beta\left(n_{t}^{*}\right) N_{t}^{*} \frac{d U^{*}}{d N}\left(N_{t+1}^{*}\right)=0 \\
& =U_{n}^{\circ}\left(n_{t}^{*}, f\left(e\left(N_{t}^{*}\right)\right), U^{*}\left(N_{t+1}^{*}\right)\right)+\beta\left(n_{t}^{*}\right) N_{t}^{*} \frac{d U^{*}}{d N}\left(N_{t+1}^{*}\right),
\end{aligned}
$$

where $U_{n}^{\circ}$ was defined in (7). For $(y, U)=\left(f\left(e\left(N_{t}^{*}\right)\right), U^{*}\left(N_{t+1}^{*}\right)\right)$, a household would set $U_{n}^{\circ}(n, y, U)=0$, so optimal and natural fertilities differ. The term $\beta\left(n_{t}^{*}\right) N_{t}^{*} \frac{d U^{*}}{d N}\left(N_{t+1}^{*}\right)$ is ignored by households; it measures the population externality in units of parent's utility.

To evaluate $\frac{d U^{*}}{d N}\left(N_{t+1}^{*}\right)$, the envelope theorem applied to (15) implies:

$$
\frac{d U^{*}}{d N}\left(N_{t+1}^{*}\right)=-u\left(c_{t+1}^{*}\right) f^{\prime}\left(e\left(N_{t+1}^{*}\right)\right) \frac{e\left(N_{t+1}^{*}\right)}{N_{t+1}^{*}}+\beta\left(n_{t+1}^{*}\right) n_{t+1}^{*} \frac{d U^{*}}{d N}\left(N_{t+2}^{*}\right)
$$

Reapplying (17) iteratively to eliminate successive future derivatives of $U^{*}$, the future terms collapse into the discounted sum

$$
\frac{d U^{*}}{d N}\left(N_{t+1}^{*}\right)=-\frac{1}{N_{t+1}^{*}} \sum_{i=1}^{\infty}\left[\prod_{j=1}^{i-1} \beta\left(n_{t+j}^{*}\right)\right] u^{\prime}\left(c_{t+i}^{*}\right) f^{\prime}\left(e\left(N_{t+i}^{*}\right)\right) e\left(N_{t+i}^{*}\right) .
$$

The infinite sum reflects the fact that a birth at $t$ creates a new dynasty whose members 
increase populations after $t+1$ and also generate externalities.

The terms $f^{\prime}\left(e_{t+i}\right) e_{t+i}$ in (18) are aggregate real population externalities measured in units of output or equivalently in units of descendants' consumption at $t+i$. Remaining terms convert the real population externalities into units of parent's (period- $t$ ) utility. The negative sign shows the externality reduces utility.

There are three interpretations of the term $f^{\prime}\left(e_{t+i}\right) e_{t+i}$. First, the market value at price $f^{\prime}$ of the emissions produced by a person born at $t+i-1$, which come at the expense of emissions by everyone else under a cap. Second, the loss of per-capita output caused by a person born at $t+i-1$ : output $f\left(e\left(N_{t+i}\right)\right)$ is lower by $f^{\prime}\left(e_{t+i}\right) e_{t+i} / N_{t+i}$; summing over the population at $t+i$ gives an aggregate loss of $f^{\prime}\left(e_{t+i}\right) e_{t+i}$. Third, the dilution of rents from auction revenue. When government auctions $\hat{E}$ permits, it receives total revenue $p_{t+i} \hat{E}=f^{\prime}\left(e_{t+i}\right) \hat{E}$ that it redistributes as equal lump sums so each person indirectly receives emission revenue $f^{\prime}\left(e_{t+i}\right) e_{t+i}$. With an additional birth at $t+i-1$, the population at $t+i$ loses the revenue $f^{\prime}\left(e_{t+i}\right) e_{t+i}$ that goes to the additional person.

Because any cap eventually binds, the discounted externality sum in $\frac{d U^{*}}{d N}\left(N_{t+1}^{*}\right)$ is strictly negative for all $t$ even if population is initially so low that $N_{t}<e^{+} E_{t}$, so the cap does not yet bind and period- $t$ permits have zero price. ${ }^{17}$ Thus starting from any population $N_{t}$, the optimal population at $t+1$ is always less than the natural population.

\section{B. Pigou Taxes on Having Children}

To compute the sequence of child taxes that would be needed to change fertility and population to optimal levels, we assume child-tax revenue is redistributed to households as equal lump sums. Let $\tau_{t}$ denote a tax per child and let $\bar{n}_{t}$ denote the average over households of $n_{t}$ in $t$, so each household pays child taxes $\tau_{t} n_{t}$ and receives lump-sum transfers of per-capita revenue $\tau_{t} \bar{n}_{t}$.

With child taxes, overall child costs include taxes and overall transfers includes lumpsum redistributions of child-tax revenue, so an individual household generically maximizes $u(w+T R-\chi n-\tau n)+\beta(n) U$ taking $w, T R=p E / N+\tau \bar{n}$, and $\tau$ as given.

${ }^{17}$ Formally: $e\left(N_{t}^{*}\right) \rightarrow e_{s s}^{*}<e^{+}$implies $f^{\prime}\left(e_{t}\right)>0$ for some $t$ so $\frac{d U^{*}}{d N}\left(N_{t+1}^{*}\right)<0$. 
The first-order condition is

$$
U_{n}^{\circ}(n, w+T R, U \mid \tau) \equiv-u^{\prime}(w+T R-\chi n-\tau n)(\chi+\tau)+\beta^{\prime}(n) U=0 .
$$

To implement the optimal population sequence, each optimal tax $\tau_{t}^{*}$ must be set so $n_{t}^{*}$, which solves (16), also solves (19). Setting $U_{n}^{*}$ from (16) equal to $U_{n}$ from (19) and noting that $w_{t}+T R_{t}-\tau_{t}^{*} n_{t}^{*}=f\left(e\left(N_{t}^{*}\right)\right)$ and $U_{t+1}=U^{*}\left(N_{t+1}^{*}\right)$ along the optimal path, $\tau_{t}^{*}$ must satisfy $u^{\prime}\left(f\left(e\left(N_{t}^{*}\right)\right)-\chi n_{t}^{*}\right) \tau_{t}^{*}=-\beta\left(n_{t}^{*}\right) N_{t}^{*} \frac{d U^{*}}{d N}\left(N_{t+1}^{*}\right)$. From (18),

$$
\begin{aligned}
\tau_{t}^{*} & =\frac{\beta\left(n_{t}^{*}\right) N_{t}^{*}}{u^{\prime}\left(c_{t}^{*}\right)}\left(-\frac{d U^{*}}{d N}\left(N_{t+1}^{*}\right)\right) \\
& =\frac{\beta\left(n_{t}^{*}\right)}{n_{t}^{*}} \sum_{i=1}^{\infty}\left[\prod_{j=1}^{i-1} \beta\left(n_{t+j}^{*}\right)\right] \frac{u^{\prime}\left(c_{t+i}^{*}\right)}{u^{\prime}\left(c_{t}^{*}\right)} f^{\prime}\left(e\left(N_{t+i}^{*}\right)\right) e\left(N_{t+i}^{*}\right) .
\end{aligned}
$$

Because the externality sum $\frac{d U^{*}}{d N}\left(N_{t+1}^{*}\right)$ is strictly negative, optimal taxes are strictly positive for all $t \geq 0$. Optimal child taxes are Pigou taxes, as in Harford (1998): the optimal tax equals the discounted present value of the externalities generated by a child and all descendants of the child. The terms in (20) other than the real externalities $f^{\prime}\left(e\left(N_{t+1}^{*}\right)\right) e\left(N_{t+1}^{*}\right)$ can be interpreted as the number of descendants in a future period times products of single-period discount factors. Overall, the $\tau_{t}^{*}$ measure population externalities in units of parent's consumption.

\section{Extensions}

We extend the model in three ways for calibrations:

\section{A. Time costs of children}

To account correctly for costs of having children, we also include time costs. This matters for the calibration because emissions restrictions that reduce $e_{t}$ also reduce the wage rate and hence the cost of having a child. The resulting boost in fertility makes it more challenging for policy to reduce fertility. 
We assume having a child requires a constant amount of parental time $\psi$ in addition to output $\chi$. Time spent having a child reduces labor supply so $l_{t}=1-\psi n_{t}$ depends on fertility, total labor supply $L_{t}=\left(1-\psi n_{t}\right) N_{t}$ differs from population $N_{t}$, and the emissions ratio depends on fertility: $e_{t}=\min \left(e^{+}, \hat{E} /\left[\left(1-\psi n_{t}\right) N_{t}\right]\right)$.

With time costs, household income depends on time cost and fertility: $y_{t}=(1-$ $\left.\psi n_{t}\right) f\left(e_{t}\right)=\left(1-\psi n_{t}\right) w_{t}+f^{\prime}\left(e_{t}\right) E_{t} / N_{t}$, which is again the sum of wage income and transfers. The marginal cost of a child becomes $\chi+\psi w_{t}$, the sum of the output cost and foregone wages. The generic household first-order condition (7) becomes $U_{n}^{\circ}(n, w, T R, U) \equiv-u^{\prime}((1-\psi n) w+T R-\chi n) \cdot(\chi+\psi w)+\beta^{\prime} U=0$, where $w=f(e)-f^{\prime}(e) e$ and $T R=f^{\prime}(e) E / N$ enter separately (not only through income). Whereas households take $w$ and $T R$ as given, optimal policy must recognize the dependence of $(w, T R)$ on fertility and population.

The changes implied by time costs carry through the dynamics and steady-state conditions. Notably, the steady-state function $S$ defined in (8) gains terms and becomes $S(n, e)=-u^{\prime}(c)(\chi+\psi w)+\beta^{\prime}(n) u(c) /[1-\beta(n)]$ where $c=(1-\psi) f(e)-\chi$ and $w=f(e)-f^{\prime}(e) e$. Roots of the resulting steady-state conditions $S\left(n^{+}, e^{+}\right)=0$, $S^{\circ}\left(e_{s s}^{\circ}\right)=S\left(1, e_{s s}^{\circ}\right)=0$, and $S^{*}\left(e_{s s}^{*}\right)=0$ exist as before. Condition (9), which ensures $n^{+}>1$, gains a time-cost term $\psi f\left(e^{+}\right)$and becomes $\chi+\psi f\left(e^{+}\right)<\phi f\left(e^{+}\right)$. The steady-state optimal and natural populations are $N_{s s}^{*}=\hat{E} /\left[(1-\psi) e_{s s}^{*}\right]$ and $N_{s s}^{\circ}=$ $\hat{E} /\left[(1-\psi) e_{s s}\right]$. As before, $e_{s s}^{\circ}<e_{s s}^{*}<e^{+}$, so $N_{s s}^{*}<N_{s s}^{\circ}$.

\section{B. Backstop Technology}

A common assumption in integrated assessment models is that a "backstop" technology may allow positive output without emissions. Backstop output is a positive value $f(0)$. If $f(0)$ is low enough, specifically, if $f(0)<f^{B} \equiv \chi /(\phi-\psi)$, then steady-state natural and optimal populations exists as above.

If $f(0)>f^{B}$, however, income loss from a cap is insufficient to reduce fertility to replacement so the population does not converge to a steady-state value. Natural fertility converges instead to the unique root $n_{s s}^{\circ}>1$ of $S\left(n_{s s}^{\circ}, 0\right)=0$, population grows without 
bound, $e_{t} \rightarrow 0$, and $f\left(e_{t}\right) \rightarrow f(0) .{ }^{18}$ Optimal fertility converges to the same limit as natural fertility: $n_{s s}^{*}=n_{s s}^{\circ}$. For all finite periods, however, optimal fertility is less than natural fertility, so the population externality exists and the optimal child tax is positive. (That is, the terms $f^{\prime}\left(e_{t+i}\right) e_{t+i}$ in (18) and in (20) are positive.) Optimal population is therefore less than optimal population at all $t$ and in the limit.

The backstop output level $f(0)$ is key to knowing the economy's fate under a cap. As long as $f(0)<f^{B}$, a cap ultimately leads to a steady state with output low enough to choke off population growth. This is true even if the cost of eliminating almost all emissions is small: fertility then would remain high so $e$ would continue to drop, until output is low enough so $\eta^{*}(N)=1$.

\section{Exogenous Growth in Factor Productivity}

To add exogenous productivity growth, we assume production is

$$
Y_{t}=F\left(L_{t} \lambda^{t}, E_{t} \alpha^{t}\right)
$$

where $\lambda \geq 1$ is an exogenously given growth factor for labor productivity and $\alpha \geq 1$ is an exogenously given growth factor for emissions productivity. Greater emissions productivity $\alpha^{t}$ means fewer emissions are needed to produce a given output from a given amount of labor.

We assume the output cost of a child grows with labor productivity $\lambda^{t}$, so the household budget becomes $c_{t}=y_{t}-\lambda^{t} \chi n_{t}$. The idea is that greater productivity means more human capital, which requires that more resources be put into each child. The assumed proportionality of the output cost of a child to $\lambda^{t}$ ensures that child costs do not vanish or explode as a fraction of income merely because productivity grows, which ensures balanced growth. ${ }^{19}$

\footnotetext{
${ }^{18}$ Because $e_{t} l_{t} N_{t}=\hat{E}$, a value $e_{t}=0$ is inconsistent with $\hat{E}>0$. Thus there is no meaningful $e_{s s}=0$, but allocations with $e_{t}>0$ in which $e_{t} \rightarrow 0$ are meaningful. In the non-generic case with $f(0)=f^{B}, e_{t} \rightarrow 0$ and $n_{t} \rightarrow 1$, so the limit condition is $S(1,0)=0$.

${ }^{19}$ Section VI.D discusses child quality and demographic transitions, which also relate to growth in child costs.
} 
An economy with growing productivity is equivalent to an economy with stationary values of growth-adjusted variables, marked with tildes. The key state variable is the growth-adjusted population $\tilde{N}_{t} \equiv N_{t} \lambda^{t} / \alpha^{t}$. Define growth-adjusted fertility $\tilde{n}_{t} \equiv n_{t} \lambda / \alpha$, which is the growth factor for $\tilde{N}_{t}$, and define costs per growth-adjusted child $\tilde{\psi} \equiv \psi \alpha / \lambda$ and $\tilde{\chi} \equiv \chi \alpha / \lambda$, so $\tilde{\psi} \tilde{n}_{t}=\psi n_{t}$ and $\tilde{\chi} \tilde{n}_{t}=\chi n_{t}$. Define growth-adjusted income $\tilde{y}_{t} \equiv$ $y_{t} / \lambda^{t}$ and emissions ratio $\tilde{e}_{t} \equiv E_{t} /\left[\left(1-\tilde{\psi} \tilde{n}_{t}\right) \tilde{N}_{t}\right]$, so $\tilde{c}_{t}=\tilde{y}_{t}-\tilde{\chi} \tilde{n}_{t}$ and $\tilde{y}_{t}=(1-$ $\left.\tilde{\psi} \tilde{n}_{t}\right) f\left(\tilde{e}_{t}\right)$. Utility $\tilde{U}_{t} \equiv U_{t} / \lambda^{(1-\theta) t}$ then satisfies the recursion (1) in growth-adjusted form: $\tilde{U}_{t}=u\left(\tilde{c}_{t}\right)+\tilde{\beta}(\tilde{n}) \tilde{U}_{t+1}$, where $\tilde{\beta}\left(\tilde{n}_{t}\right) \equiv \lambda^{(1-\theta)} \beta\left(\tilde{n}_{t} \alpha / \lambda\right)=\lambda^{(1-\theta)} \beta\left(n_{t}\right)$. Hence the problem of maximizing $\tilde{V}_{t}=\tilde{\beta}\left(\tilde{N}_{t}\right) \tilde{U}_{t}$ by choice of $\left(\tilde{N}_{t}, E_{t}\right)$ has the same form as the problem without productivity growth, except that growth-adjusted (tilde) variables replace regular variables. All analysis from the previous sections goes through with growth-adjusted variables and parameters replacing regular variables and parameters.

In the no-cap era, the emissions ratio is $\tilde{e}_{t}=e^{+}$and household income follows $y_{t}^{+} \equiv$ $\left(1-\tilde{\psi} \tilde{n}_{t}\right) f\left(e^{+}\right) \lambda^{t}$. A perfect-foresight solution is pair $\left(\tilde{n}^{+}, \tilde{U}^{+}\right)$with $\tilde{U}^{+}=u((1-$ $\left.\left.\tilde{\psi} \tilde{n}^{+}\right) f\left(e^{+}\right)-\tilde{\chi} \tilde{n}^{+}\right) /\left(1-\tilde{\beta}\left(\tilde{n}^{+}\right)\right)$, where $\tilde{n}^{+}$is optimal given $\tilde{U}^{+}$. In any solution, growthadjusted population grows at rate $\tilde{n}^{+}-1$. Because $E_{t}=\tilde{e}_{t}\left(1-\tilde{\psi} \tilde{n}^{+}\right) \tilde{N}_{t}$ and $\tilde{e}_{t}=e^{+}$, emissions also grow at rate $\tilde{n}^{+}-1$. We assume $\tilde{\chi}+\tilde{\psi} f\left(e^{+}\right)<\tilde{\phi} f\left(e^{+}\right)$where $\tilde{\phi}=$ $1 /[1+\omega(1-\tilde{\beta}(1)) / \tilde{\beta}(1)]<1$, so $\tilde{n}^{+}>1 .^{20}$

In the cap era, growth-adjusted optimal population converges to a steady-state value $\tilde{N}_{s s}^{*}$. Unless $\alpha=\lambda$, actual (non-growth-adjusted) population changes over time when growth-adjusted population is constant in steady state. Specifically, $\tilde{n}_{s s}^{*}=n_{s s}^{*} \lambda / \alpha=1$ implies $n_{s s}^{*}=\alpha / \lambda$. This is a balanced-growth condition. ${ }^{21}$ Growth in labor productivity $(\lambda)$ introduces an increasing trend in each person's emissions footprint and growth in emissions productivity $(\alpha)$ introduces a decreasing trend, so exogenous productivity growth overall introduces per-capita emissions growth with factor $\lambda / \alpha$ per period. To

\footnotetext{
${ }^{20}$ Emissions have increased historically, consistent with $\tilde{n}^{+}>1$. If future fertility were to fall sufficiently due to changes in tastes or if $\alpha / \lambda$ were to fall sufficiently, then $\tilde{n}^{+}$could fall below one. Then emissions and the emissions problem would eventually vanish.

${ }^{21}$ The production function (21) implies that output growth arises from growth in the inputs $l_{t} N_{t} \lambda^{t}$ and $E_{t} \alpha^{t}$. In steady state with actual fertility constant at $n_{s s}$, effective labor $\left(1-\psi n_{s s}\right) N_{t} \lambda^{t}$ has growth factor $n_{s s} \lambda$. Because emissions are capped at $\hat{E}$, the input $E_{t} \alpha^{t}$ has growth factor $\alpha$. Balanced growth requires $n_{s s} \lambda=\alpha$, or $n_{s s}=\alpha / \lambda$.
} 
hold total emissions constant in steady state, population must therefore grow with factor $\alpha / \lambda$. By analogous reasoning, steady-state natural fertility is also $n_{s s}^{\circ}=\alpha / \lambda$.

Four growth factors describe steady states in the cap era. The steady-state optimal and natural populations grow with factor $\alpha / \lambda$, as just noted. Outputs per person and living standards grow with factor $\lambda$ because $\tilde{y}_{s s}^{*}=y_{t}^{*} / \lambda^{t}$ and $\tilde{y}_{s s}^{\circ}=y_{t}^{\circ} / \lambda^{t}$ are constant. Total outputs, the products of population and output per person, grow with factor $\alpha$, the product of $\alpha / \lambda$ and $\lambda$. Finally total emissions are constant, as output grows with the emissions-productivity growth factor $\alpha$. The outcome is Malthusian modified for productivity growth: living standards continue to grow as long as $\lambda>1$; total outputs continues to grow as long as $\alpha>1$; and population grows (or shrinks) unless $\alpha=\lambda$.

Taxes in the transformed economy, $\tilde{\tau}_{t} \equiv \tau_{t}(\alpha / \lambda) / \lambda^{t}$, are taxes per growth-adjusted child scaled by productivity growth. To convert optimal taxes $\tilde{\tau}_{t}^{*}$ to taxes per actual child $\tau_{t}^{*}$, we divide out the adjustment factor $(\alpha / \lambda) / \lambda^{t}$. The actual tax grows with factor $\lambda$, as does actual income along the optimal path, $y_{t}^{*} \equiv\left(1-\tilde{\psi} \tilde{n}_{t}^{*}\right) f\left(\tilde{e}_{t}^{*}\right) \lambda^{t}$. In the calibrations below we remove growth factors by reporting optimal taxes as shares of income ${ }^{22}$

$$
\{\tau / y\}_{t}^{*} \equiv \frac{\tau_{t}^{*}}{y_{t}^{*}}=\frac{\tilde{\tau}_{t}^{*} \lambda / \alpha}{\left(1-\tilde{\psi} \tilde{n}_{t}^{*}\right) f\left(\tilde{e}_{t}^{*}\right)} .
$$

\section{Calibration}

We calibrate the model to a growing world economy with annual steady-state population growth of 1.4 percent, per-capita output growth of 1.7 percent, and aggregate emissions growth of 1.8 percent, which were actual world rates over 1990-2005. ${ }^{23}$ A period equals 30 years, so $n^{+}=1.52, \tilde{n}^{+}=n^{+} \lambda / \alpha=1.72, \alpha=1.48$, and $\lambda=1.67{ }^{24}$ The value of $b_{0}$ is chosen so the household's first-order condition holds given these growth

\footnotetext{
${ }^{22}$ To interpret $\{\tau / y\}_{t}^{*}$ in a real world with two-adult households, each parent can be seen as paying one-half of the tax so the tax on any single child as a share of household income is $\{\tau / y\}_{t}^{*} / 2$. For the couple to replace themselves takes two children, so each would pay $\{\tau / y\}_{t}^{*}$ for replacement.

${ }^{23}$ see World Resources Institute (2008). Trend assumptions matter-see sensitivity analysis below.

${ }^{24}$ Specifically $n^{+}=\exp (30 \cdot .014)=1.52$. Per-capita income grows with factor $\lambda$ so $\lambda=\exp (30 \cdot .017)=1.67$. Total emissions grow at the same rate as productivity-adjusted population, so $\tilde{n}^{+}=n^{+} \lambda / \alpha=\exp (30 \cdot .018)=1.72$. This implies $\alpha=n^{+} \lambda / \tilde{n}^{+}=1.48$.
} 
rates. We choose units so $\tilde{N}_{0}=N_{0}=1, f\left(e^{+}\right)=1$, and $e^{+}=1$. Unless noted, all variables except child taxes are growth-adjusted.

We consider two production functions. Cobb-Douglas production is $f(\tilde{e})=f_{0} \tilde{e}^{f_{1}}\left(f_{2}-\right.$ $\tilde{e})^{1-f_{1}}$ where $f_{0}, f_{1} \in(0,1)$, and $f_{2}$ are parameters. ${ }^{25}$ With Cobb-Douglas production, the factor share of emissions rises monotonically from zero at $e^{+}$to $f_{1}$ as $\tilde{e}$ falls to zero. For any $f_{1}$, units choices pin down $f_{0}$ and $f_{2}: f^{\prime}\left(e^{+}\right)=0$ implies $f_{2}=e^{+} / f_{1}$, and $f\left(e^{+}\right)=1$ implies $f_{0}=\left[f_{1} /\left(1-f_{1}\right)\right]^{1-f_{1}} / e^{+}$. To set $f_{1}$, we assume it costs 3 percent of output to reduce emissions by 25 percent, so $f\left(0.75 \cdot e^{+}\right)=0.97$. This implies $f_{1}=0.483$. A 3-percent cost is in the range of estimates in Stern (2007). We also evaluate a 2-percent cost below, with $f_{1}=0.371$.

Cobb-Douglas production does not allow a positive backstop. To study a backstop and get a sense of how sensitive results are to the form of production, we also consider the abatement-cost specification used in many integrated assessment models: $f(\tilde{e})=1-$ $\left(1-g_{0}\right)\left(1-\tilde{e} / e^{+}\right)^{g_{1}}$, where $g_{0}$ and $g_{1}$ are parameters and backstop output is $f(0)=g_{0} \cdot{ }^{26}$ When we assume no backstop $\left(g_{0}=0\right)$, we set $g_{1}$ by again assuming it costs 3 percent of output to reduce emissions by 25 percent, so $g_{1}=3.32$. With a positive backstop, we leave the curvature $g_{1}$ unchanged and simply assume a positive $g_{0}$, which proportionately reduces abatement costs at any $\tilde{e}$.

We assume children have an output cost of $\chi=0.138$ and a time cost of $\psi=0.110$, which are based on U.S. data. The output cost is from the sum of expenditures by families on children plus expenditures on K-12 and college education. The time cost assumes the difference between male and female labor-force participation rates is due solely to time devoted to having children so that with zero children, the average participation rate would equal the current male rate (0.76) instead of the current average of male and female rates

\footnotetext{
${ }^{25}$ The form may be derived from three primitive assumptions: (i) labor is used to produce two intermediate goods in amounts $y_{1}$ and $y_{2}$ according to the linear technology $y_{1}+y_{2}=f_{2} ;$ (ii) a unit of good 1 generates a unit of emissions so $\tilde{e}=y_{1}$, whereas good 2 generates no emissions; and (iii) output per unit of labor is a Cobb-Douglas function $f_{0} y_{1}^{f_{1}} y_{2}^{1-f_{1}}$ (If output per unit of labor were a CES function of $y_{1}$ and $y_{2}$ with an elasticity other than one, the factor share of emissions would approach either zero or one as $\tilde{e} \rightarrow 0$, which may be undesirable to impose.)

${ }^{26} \mathrm{An}$ interpretation is that a unit of labor gives a unit of output and a unit of emissions if no resources are devoted to abatement, and the cost of abating $1-\tilde{e}$ units of emissions is $\left(1-g_{0}\right)(1-\tilde{e})^{g_{1}}$ units of output. The factor share of emissions in abatement-cost cases has a knife-edge, which partly motivates why we use Cobb-Douglas for most calibrations. Without a backstop, the factor share rises monotonically from zero at $\tilde{e}=1$ to one at $\tilde{e}=0$, but with any positive backstop, the factor share rises from zero at $\tilde{e}=1$ to a peak, then falls to zero at $\tilde{e}=0$.
} 
TABle 1-STEAdy States

\begin{tabular}{|c|c|c|c|c|c|c|c|}
\hline Case & $\begin{array}{c}\text { Population } \\
\text { Policy }\end{array}$ & $\tilde{N}_{s s}$ & $\tilde{y}_{s s}$ & $\frac{\tilde{e}_{s s}}{e^{+}}$ & $N_{\text {peak }}$ & $\frac{f^{\prime} \tilde{e}_{s s}}{f}$ & $\{\tau / y\}_{s}^{*}$ \\
\hline \multirow[t]{2}{*}{ 1. base $(\theta=b=0.8)$} & none & 9.16 & 0.408 & 0.101 & 3.76 & 0.457 & \\
\hline & optimal & 2.39 & 0.721 & 0.386 & 1.28 & 0.365 & 0.211 \\
\hline \multirow[t]{2}{*}{ 2. $\theta=b=2$} & none & 44.1 & 0.195 & 0.021 & 13.9 & 0.478 & \\
\hline & optimal & 11.1 & 0.373 & 0.083 & 3.52 & 0.469 & 0.957 \\
\hline \multirow[t]{2}{*}{ 3. $\theta=0.95, b=0.8$} & none & 9.90 & 0.394 & 0.093 & 4.16 & 0.459 & \\
\hline & optimal & 7.32 & 0.452 & 0.126 & 3.12 & 0.450 & 0.068 \\
\hline \multirow[t]{2}{*}{ 4. $f(0.75)=0.98$} & none & 15.6 & 0.418 & 0.059 & 4.96 & 0.357 & \\
\hline & optimal & 3.96 & 0.665 & 0.233 & 1.63 & 0.311 & 0.180 \\
\hline \multirow[t]{2}{*}{ 5. $n^{+}=1$} & none & 3.57 & 0.633 & 0.277 & 1.17 & 0.404 & \\
\hline & optimal & 1.20 & 0.889 & 0.825 & 1.00 & 0.141 & 0.051 \\
\hline
\end{tabular}

(0.685). Details are in the appendix.

The time cost implies that per-capita labor in the no-cap era is $1-\tilde{\psi} \tilde{n}^{+}=0.833$, per-capita income is $\tilde{y}^{+}=\left(1-\tilde{\psi} \tilde{n}^{+}\right) f\left(e^{+}\right)=0.833$, and uncapped emissions at $t=0$ are $E_{0}^{+} \equiv e^{+}\left(1-\tilde{\psi} \tilde{n}^{+}\right) \tilde{N}_{0}=0.833$.

\section{A. Base Case}

The base case assumes that the scientific knowledge arriving at $t=0$ is that an emissions freeze is necessary. A freeze means setting $\hat{E}=E_{0}^{+}=0.833$. A reasonable range of estimated $\theta$ values is $0.5-5.0$ (see e.g. Ogaki and Reinhardt, 1998; Bansal and Yaron, 2004). As base case, we set $\theta=b=0.8$, and we assume Cobb-Douglas production.

Steady-state results for the base case and several alternatives are in table 1 . In the base case, the growth-adjusted optimal population in steady-state is 2.39 times $\tilde{N}_{0}$, the steadystate emissions ratio is 0.386 times the emissions ratio without a cap, and steady-state output per-capita is 0.721 , which is 13.5 percent less than $\tilde{y}^{+}=0.833$. The growthadjusted natural population in steady state is 9.16 , or almost four $(9.16 / 2.39)$ times the optimal population. The emissions ratio of 0.101 and the per-capita output of 0.408 are substantially less than the optimal values.

The fertility functions (figure 3 ) imply that the optimal and natural populations con- 
verge smoothly from $\tilde{N}_{0}=1$ to the steady-state values. ${ }^{27}$ After five generations, $\tilde{N}_{5}^{\circ}=$ 6.84 , for instance, and after ten generations, $\tilde{N}_{10}^{\circ}=8.97$, close to the steady-state value of 9.16 .

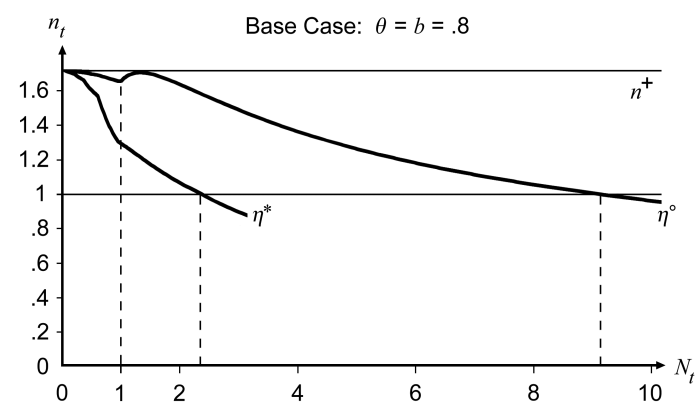

Figure 3. Base Case Fertilities

Figure 4 shows the actual (not adjusted) optimal and natural populations. In the no-cap era, population increases exponentially to infinity. With a cap, the optimal and natural populations grow at rate $(\alpha-\lambda) / \lambda=-0.113$ in steady state so both actual populations peak after a cap is imposed and then fall. World population under a cap would peak at 1.28 times its current level under the optimal population policy, but at 3.76 times its current level along the natural path, as reported in column $N_{\text {peak }}$ in table 1.

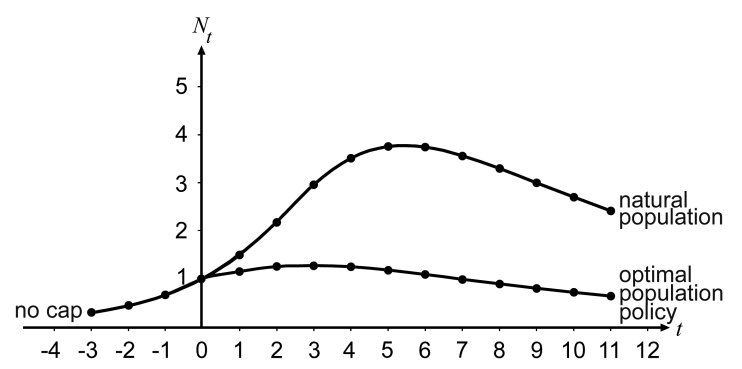

Figure 4. Populations

Permit revenue is 36.5 percent of output in the optimal steady state and 45.7 percent of output in the natural steady state. These numbers are quite large compared with the Federal spending share over 1990-2005, which averaged about 20 percent of output. An intuition for the large revenue comes from figure 1. Population growth under a cap drives

\footnotetext{
${ }^{27}$ The appendix describes the numerical procedures. The positively sloped segment of $\eta^{\circ}$ at $N$ slighly above one is due to the calibrated time cost of children (which is not present in figure 2): as the cap begins to bind for $N>1$, a lower wage reduces the cost of children.
} 
down emissions per capita $e$, which reduces per-capita income and hence fertility. Permit revenue is $f^{\prime}(e) e$ in figure 1. Revenue is small right after the cap is imposed (e.g., 4.2 percent of output at $t=0$ under the optimal policy), but increases as the emissions ratio falls below one. The large revenue numbers in steady state indicate that the process must go a long way for the economy to get to a new steady state. ${ }^{28}$ Steady-state revenue is greater in the natural than the optimal steady state because the emissions ratio declines further $\left(e_{s s}^{0}=0.101\right.$ versus $\left.e_{s s}^{*}=0.386\right)$.

The optimal child tax in steady state is 21.1 percent of per-capita income. ${ }^{29}$ To get a sense of this number in the U.S. context, U.S. personal income is roughly $\$ 55,000$ per adult per year, which may be interpreted as uncapped income $\left(\tilde{y}^{+}\right)$measured in dollars per year. Steady-state optimal income $\left(\tilde{y}_{s s}^{*}\right)$, which is 13.5 percent less than uncapped income, would then be about $\$ 48,000$. Thus a child tax of 21.1 percent is equivalent to a tax of about $\$ 10,000$ each year for 30 years (the length of a generation in the model) for each child. ${ }^{30}$ An alternative sense is that the annual cost of a child is about $\$ 13,000$, of which about $\$ 6,000$ is time costs. The optimal child tax therefore raises the full cost of a child in steady state by about three-quarters, from $\$ 13,000$ to $\$ 23,000$, to just under half $(\$ 23,000 / \$ 48,000)$ of income.

The time path of the optimal child tax in the base case is shown in row 1 of table 2 . The optimal tax in the transition period $t=0$ is substantially less than in steady state. The base case assumes that uncapped emissions at $t=0$ just equal the optimal cap, $E_{0}^{+}=\hat{E}$. If information arrived instead that $\hat{E}$ is greater or less than $E_{0}^{+}$, steady state values for $\tilde{y}_{s s}$,

\footnotetext{
${ }^{28}$ As can be seen from figure 1, revenue would not be substantial in only two cases: (i) if the slope $f^{\prime}$ is always small and there is a high backstop output, so the entire emissions problem could be eliminated at little cost by simply restricting emissions to zero; or (ii) if $f^{\prime}$ is small until $e$ is close to zero, at which point $f$ bends sharply downward to a zero backstop, so almost all emissions could be eliminated at little cost. It may be difficult to distinguish the two cases from current economic observations around $e=e^{+}$; but neither case is of interest under the maintained hypothesis that emissions are a serious problem. (In the second case, emissions are a problem only in the future when population has increased enough so incomes are low.)

${ }^{29}$ Income excludes redistributions of child-tax revenue, so a tax of 21.1 percent of income is equivalent to a tax of 17.4 $(=21.1 / 1.211)$ percent of income plus redistributions of child-tax revenue.

${ }^{30}$ Kelly and Kolstad (2001) calculate welfare costs from a marginal child in the range \$200-\$800. Such costs are tiny compared with costs of $\$ 10,000$ per year for 30 years. Kelly and Kolstad implicitly assume a backstop output of $f(0)=.93$ and also assume that population grows at an exogenously given rate that itself decreases at an exogenously given rate. With their production function in our model, the optimal policy would be to drive the emissions ratio to zero in steady state. This does not happen in their calculations because they assume growth slows enough so the backstop is never reached.
} 
TABle 2-Time Paths of Optimal Child Taxes

\begin{tabular}{lcccccc}
\multicolumn{1}{c}{ Case } & $\{\tau / y\}_{0}^{*}$ & $\{\tau / y\}_{1}^{*}$ & $\{\tau / y\}_{2}^{*}$ & $\{\tau / y\}_{3}^{*}$ & $\{\tau / y\}_{4}^{*}$ & $\{\tau / y\}_{s s}^{*}$ \\
\hline base case: $\hat{E} / E_{0}^{+}=1$ & 0.110 & 0.143 & 0.166 & 0.181 & 0.192 & 0.211 \\
25 percent cut: $\hat{E} / E_{0}^{+}=0.75$ & 0.147 & 0.168 & 0.183 & 0.193 & 0.199 & 0.211 \\
25 percent slack: $\hat{E} / E_{0}^{+}=1.25$ & 0.076 & 0.125 & 0.154 & 0.173 & 0.186 & 0.211 \\
abatement cost, $f(0)=0$ & 0.130 & 0.184 & 0.216 & 0.233 & 0.242 & 0.249 \\
abatement cost, $f(0)=0.4$ & 0.094 & 0.134 & 0.158 & 0.173 & 0.183 & 0.201 \\
abatement cost, $f(0)=0.6$ & 0.067 & 0.090 & 0.097 & 0.096 & 0.090 & 0 \\
\hline
\end{tabular}

$\tilde{e}_{s s}, f^{\prime} \tilde{e}_{s s} / f$, and $\{\tau / y\}_{s s}^{*}$, would not change, but $\tilde{N}_{s s} / N_{0}$ would be proportionally higher or lower, and the transition paths would differ. ${ }^{31}$ Notably, the path of optimal child taxes would differ, as shown in rows 2 and 3 of table 2 . If $\hat{E} / E_{0}^{+}=0.75$, so emissions must be cut at $t=0$, the optimal policy response is to reduce fertility more aggressively by setting child taxes higher than under the freeze. If $\hat{E} / E_{0}^{+}=1.25$, emission can still grow and optimal taxes are initially less than under the freeze. In all cases, child taxes converge to the steady value of 21.1 percent.

\section{B. Sensitivity Analysis-Utility Curvatures}

Table 1 also reports sensitivity analysis of individual base-case assumptions. Jones, Schoenbroodt, and Tertilt (2008) and Jones and Schoonbroodt (2010) argue that $(\theta>$ $1, b>1)$ explains historical fertility trends better than $(\theta<1, b<1)$, so the first sensitivity test assumes $\theta=b=2$ (case 2). Steady-state populations are then substantially greater, income is lower, and optimal child taxes are higher than in the base case. In steady state, optimal population is 11.1 , income is $\tilde{y}_{s s}^{*}=0.373$, and the child tax is 95.7 percent of income (which is 48.9 percent of income plus redistributions of child-tax revenue, $0.957 / 1.957)$. The ratio of steady-state natural to optimal population is still about four $(44.1 / 11.1)$,

\footnotetext{
${ }^{31}$ There are two interpretations of $\hat{E} / E_{0}^{+}$. First, information about a given $\hat{E}$ may arrive earlier or later than assumed in the base case. If time is re-indexed so information arrives at $t=0$, earlier arrival of information means $N_{0}<1$ and hence $E_{0}^{+}<\hat{E}$. Second, for given $E_{0}^{+}$, one may consider alternative (hypothetical) scientific news that reveal higher or lower $\hat{E}$. In all cases the dynamics depend on the ratio $\hat{E} / E_{0}^{+}$.
} 
Assuming high values of $\theta$ and $b$ might make sense if one believes fertility is insensitive to economic incentives, but this is a pessimistic assumption here. The fertility path in figure 5 makes clear why. A cap reduces children's utility, and when $b>1$, this causes a burst in natural fertility. As a result, a greater income fall and hence a greater population are needed to reduce fertility to replacement. Moreover, the wide gap between natural and optimal fertility implies that high child taxes are needed to implement the optimal fertility.

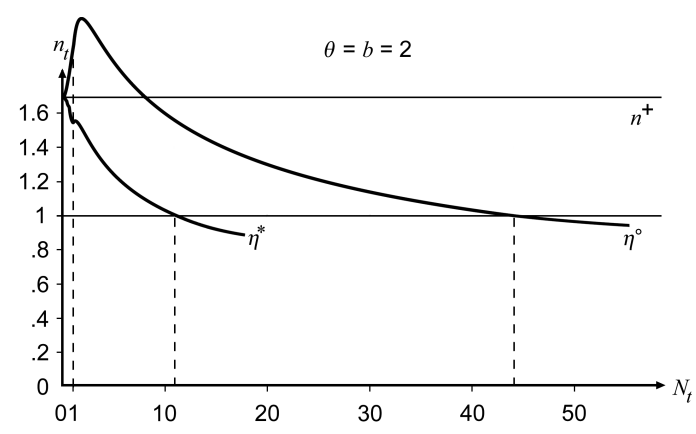

Figure 5. Dynamics when $\theta=b=2$.

We also consider the equal-curvatures assumption, $\omega=1$. Because $\omega$ measures how much parents care about per-child consumption relative to the number of children, and the population externality is a loss from lower per-child consumption, a lower $\omega$ tends to reduce the utility value of the externality. Thus real reductions in children's utility and the real population externality might be substantial, but with low $\omega$, parents would simply not care much about this so the gap between natural and optimal population would be small. To judge this, we modify the baseline case, assuming $\omega=0.25$. When $\theta=$ 0.95 and $b=0.8$ (case 3 ), the ratio of natural to optimal steady-state population is 1.4 and the steady-state child tax is 6.8 percent.

The population externality thus tends to be substantial because empirically reasonable assumptions about utility curvature imply that the desire to have children remains strong as $e_{t}$ falls below $e^{+}$, so $e_{t}$ ends up being driven a fair bit below $e^{+}$. 


\section{Sensitivity Analysis-Technology}

Optimal policy depends on the cost of reducing emissions. Table 1 (case 4) reports steady-state results when $f$ is parameterized assuming it costs 2 percent of output to reduce emissions by 25 percent $(f(0.75)=0.98)$, instead of 3 percent as in the base case. This reduces the population externality but not greatly: the optimal child tax falls from 21.1 percent in the base case to 18.0 percent. The reason is that when it is less costly to reduce emissions, incomes and hence fertility are higher at any given population, and steady state is reached only when population is so high and the emissions ratio so low that incomes are close to incomes in the base case. That is, the income reductions needed to choke off population growth doesn't change much when the output cost of reducing emissions falls. ${ }^{32}$

We also examine the abatement-cost specification. Under base-case utility assumptions, the critical backstop is $f^{B}=0.507$ so we consider backstops of $0,0.4$, and 0.6 . Comparing the first two rows of table 3 with the first two rows of table 1 shows the effects of changing from a Cobb-Douglas to an abatement-cost specification with no backstop. Steady-state natural and optimal populations fall by a bit less than half and factor shares of emissions rise, but optimal child taxes change little, rising from 21.1 percent to 24.9 percent of income. Comparing rows of table 3, a higher backstop means smaller income reductions as well as lower population externalities, but the effect is not great as long as $f(0)<0.507$ (so growth-adjusted population is constant in steady state). From table 3 , the ratio of the natural to the optimal steady-state populations varies between about three and five when $f(0)<0.507$.

If $f(0)>0.507$, adjusted population grows forever and the population externality vanishes in the limit, quite a different long-run outcome than when $f(0)<0.507$. The paths of the economy for the first few periods after transition, however, can be similar.

\footnotetext{
${ }^{32}$ In detail, steady-state natural income is slightly higher than in the base case, 0.418 instead of 0.408 , but steady-state optimal income is lower, 0.665 instead of 0.721 . The smaller difference between $\tilde{y}_{s s}^{*}$ and $\tilde{y}_{s s}$ means the real externality terms $f^{\prime}(e) e$ in (18) are lower. Steady-state emission ratios are much reduced: $\tilde{e}_{s s}^{\circ}$ is only 0.059 instead of 0.101 in the base case, and $\tilde{e}_{s s}^{*}$ is 0.233 instead of 0.386 . Consequently, $\tilde{N}_{s s}^{\circ}$ and $\tilde{N}_{s s}^{*}$ are roughly two-thirds greater than in the base case, but their ratio remains about four.
} 


\begin{tabular}{cccccccc}
\multicolumn{7}{c}{ Table 3-Steady States with Abatement-Cost Production } \\
Case & $\begin{array}{c}\text { Population } \\
\text { Policy }\end{array}$ & $\tilde{N}_{s s}$ & $\tilde{y}_{s s}$ & $\frac{\tilde{e}_{s s}}{e^{+}}$ & $N_{\text {peak }}$ & $\frac{f^{\prime} \tilde{e}_{s s}}{f}$ & $\{\tau / y\}_{s s}^{*}$ \\
\hline$f(0)=0$ & none & 4.84 & 0.374 & 0.190 & 2.85 & 0.842 & \\
& optimal & 1.58 & 0.805 & 0.586 & 1.09 & 0.431 & 0.249 \\
$f(0)=0.4$ & none & 15.6 & 0.438 & 0.059 & 4.33 & 0.168 & \\
& optimal & 2.84 & 0.702 & 0.325 & 1.36 & 0.348 & 0.201 \\
$f(0)=0.6$ & none & $\infty$ & 0.532 & 0 & $\infty$ & 0 & \\
& optimal & $\infty$ & 0.532 & 0 & $\infty$ & 0 & 0 \\
\hline
\end{tabular}

Table 2 shows this. With $f(0)=0.6$, abatement costs at any $\tilde{e}$ are reduced by 60 percent compared with abatement costs with $f(0)=0$, and the optimal child tax in the transition period is similarly about half of the tax when $f(0)=0$. With $f(0)=0.6$, the tax peaks in the second period after transition at almost 10 percent, which is still about half the tax in the base case, then goes to zero in steady state. ${ }^{33}$

\section{Sensitivity Analysis-Trend Assumptions}

An important possibility is that birth rates may decline due to demographic transitions unrelated to the environment. Becker (1960) views fertility as a mix of two variables, child quantity and child quality, and the demographic transition as a shift from a highquantity/low-quality mix to a low-quantity/high-quality one. Subsequent literature has proposed a number of mechanisms for the shift, some stressing effects of mass education (Caldwell, 1980, 1982). Studying the case of countries in demographic transition by taking a stand on the details behind the shift and adding a full model of the demographic transition is beyond the scope of the current paper. However, it is easy to alter the trend assumptions of the model to evaluate alternative assumptions about demographic and productivity trends.

The maintained hypothesis that emissions are a problem requires $\tilde{n}^{+}=n^{+} \lambda / \alpha>1$ so emissions tend to rise on their own. Broadly, changes in trend assumptions that lower $\tilde{n}^{+}$

\footnotetext{
${ }^{33}$ In the case with $f(0)=0.6$, both $\tilde{N}_{s s}$ and $\tilde{N}_{s s}^{*}$ go to infinity but their ratio converges to about 3.4. Also, population growth remains positive as the emissions ratio converges to zero: $\tilde{n}_{t}$ converges to 1.16 and actual (not adjusted) population growth $n_{t}=\tilde{n}_{t}(\alpha / \lambda)$ converges to 1.03 . In the limit, $n_{t}^{*}$ converges to the same limit of 1.03 . This is substantially lower than population growth of $n^{+}=1.52$ in the uncapped economy.
} 
mean less pressure from emissions growth, which reduces the population externality.

In the base case the underlying trends in population and technology growth equal actual values over $1990-2005, n^{+}=1.52$ and $\lambda / \alpha=1.13$, so $\tilde{n}^{+}=1.72$. To evaluate a world in which all countries have undergone a demographic transition, we recalibrate the model to the replacement value $n^{+}=1$, so world population would remain constant absent policy. Then $\tilde{n}^{+}=1.13$, as the only force driving emissions growth is technological change in a person's carbon footprint $\lambda / \alpha$. In table 1 (case 5), the implied population externality is 5.1 percent of parental income per child, lower than in the base case but not negligible. ${ }^{34}$

Because $\tilde{n}^{+}=n^{+} \lambda / \alpha$, the same 5.1 percent steady-state externality would arise if assumed fertility $\left(n^{+}\right)$were left at the base case value of 1.52 but the environmental productivity-growth factor $\alpha$ were instead assumed to increase permanently from 1.48 to $2.25=1.48 \cdot 1.52$.

\section{E. Sensitivity to Environmental Assumptions}

This section examines how results differ when S1-S3 are relaxed and greenhouse gas stocks enter as state variable. The goal is to explore the sensitivity of previous results to slight generalizations of the model. Modeling of the environment in detail is beyond the scope of the paper.

We follow integrated assessment models in assuming that damages are caused by atmospheric temperatures $\left(X^{T}\right)$, which are caused by greenhouse gas stocks $\left(X^{G}\right)$ that accumulate over time from emissions, and that damages have the functional form $\delta_{t}=$ $\kappa\left(X_{t}^{T}\right) /\left(1+\kappa\left(X_{t}^{T}\right)\right)$ with quadratic $\kappa\left(X_{t}^{T}\right)=\kappa_{0} \cdot\left(X_{t}^{T}\right)^{2}, \kappa_{0}>0$ (e.g., see Nordhaus and Boyer 2000). We model $X_{t}^{T}=\lambda \log \left(1+X_{t}^{G}\right) / \log (2)$ as logarithmic with temperature sensitivity parameter $\lambda>0$ and $X_{t+1}^{G}=\gamma X_{t}^{G}+E_{t}$ as linear with parameter $\gamma \in(0,1){ }^{35}$

\footnotetext{
${ }^{34}$ This scenario is also extreme in two ways: (i) it assumes poor countries reduce fertility to replacement without any increase in incomes and hence emissions; and (ii) it ignores other sources of population growth such as changes in mortality. The United Nations (2010) median-fertility population projection assumes fertility in all countries is close to replacement, and finds that world population should nonetheless continue to grow until about 2100.

${ }^{35}$ Temperature $X^{T}$ is measured in degrees Celsius and $X^{G}$ as greenhouse gas concentration above pre-industrial level. The unit coefficient on $E$ is a normalization. A logarithmic form linking radiative forcing (temperature) to greenhouse gas stocks is a standard assumption. We simplify by abstracting from dynamics in this relationship and from interactions
} 
Combining these assumptions, the environmental state is univariate, $X_{t}=\mathbf{X}_{t}=X_{t}^{G}$, and the damage function is:

$$
\delta_{t}=\delta\left(X_{t}\right)=\frac{\kappa\left[\frac{\lambda}{\log (2)} \log \left(1+X_{t}\right)\right]}{1+\kappa\left[\frac{\lambda}{\log (2)} \log \left(1+X_{t}\right)\right]} .
$$

We calibrate $\gamma=0.75, \lambda=3$, and $\kappa_{0}=7.7 \cdot 10^{-4} .36$

Pindyck (2013) argues that models with quadratic costs and constant temperature sensitivity are inadequate for modeling damages at high greenhouse gas levels. This is important here because (calibrated) human preferences for children are strong, and hence a threat of high damages is needed to trigger damage-avoiding policies. Hence we interpret the regime shift at $t=0$ as discovery of a critical range $\mathcal{X}$ in which damages are greater than under (22). ${ }^{37}$ We model this critical range numerically in three versions that relax S1-S3 successively.

First we replace S3 by (22) but maintain S1-S2; that is, (22) applies only for $E_{t} \leq \hat{E}$. Solving the general Bellman equation (4) then yields $E_{t}^{*}=E_{t}^{\circ}=\min \left\{e^{+} \tilde{L}_{t}, \hat{E}\right\}$, where $\tilde{L}_{t}=\left(1-\tilde{\psi} \tilde{n}_{t}\right) \tilde{N}_{t}$ accounts for growth and time costs of children. That is, a cap at $\hat{E}$ is still optimal.

Initial values require care because $X^{G}$ is a physical variable: current greenhouse gas stocks are about 40 percent above pre-industrial levels, so $X_{0}=0.40$ is a given. For $t \leq 0$, uncapped emissions grow at rate $\tilde{n}^{+}-1 .{ }^{38}$ Hence $X_{t}=\sum_{i \geq 1} \gamma^{i-1} E_{t-i}=$ $e^{+} \tilde{L}_{t} /\left(\tilde{n}^{+}-\gamma\right)$, so $X_{t}=\left(1-\tilde{\psi} \tilde{n}^{+}\right) e^{+} \tilde{N}_{t} /\left(\tilde{n}^{+}-\gamma\right)$ for $t \leq 0$ is proportional to population. To reach $X_{0}=0.40$ at $t=0$ it must be that $e^{+} \tilde{N}_{0}=0.464$. To normalize $\tilde{N}_{0}=1$,

${ }^{36}$ Setting $\gamma=0.75 \approx 0.9^{3}$ converts an estimated persistence of 0.9 per decade (e.g. Kelly-Kolstad 2001) to 30-year, generational frequency; $\lambda=3$ is fairly standard (as round number); $\kappa_{0}=0.00077$ is calibrated so $\delta$ matches Nordhaus and Boyer's (2000, Table 4-10) estimate that non-catastrophic damages are $0.48 \%$ of output at 2.5 degree warming, Calibrations with reasonably higher or lower values yield similar results.

${ }^{37}$ Earlier work also noted a role for catastrophic damages. In Nordhaus and Boyer (2000), for example, the expected value of possible catastrophic damage is more than twice the non-catastrophic cost $(1.02 \%$ vs. $0.48 \%)$. We follow Pindyck in modeling catastrophe separately,

${ }^{38}$ This involves a slight approximation because $\delta_{t}>0$ implies reduced output, which affects fertility even if households do not recognize the cause of reduced output. However, $\delta\left(X_{t}\right)$ for $t<0$ (so $X_{t}<0.4$ ) is so small that we calibrate $\tilde{n}^{+}$as before.
} 
TABle 4-Steady States With Non-Zero Damages

\begin{tabular}{cccccccccc}
\multicolumn{2}{c}{ Case } & $\begin{array}{c}\text { Population } \\
\text { Policy }\end{array}$ & $E_{s s}$ & $X_{s s}$ & $\delta_{s s}$ & $\tilde{N}_{s s}$ & $\frac{\tilde{e}_{s s}}{e^{+}}$ & $\tilde{y}_{s s}$ & $\frac{f^{\prime} \tilde{e}}{f}$ \\
\hline$\delta 1$. & cap $\hat{E}$ & none & 0.386 & 1.55 & 0.012 & 8.91 & 0.104 & 0.408 & 0.456 \\
& & optimal & 0.386 & 1.55 & 0.012 & 2.34 & 0.395 & 0.717 & 0.362 \\
$\delta 2$. & cap $\hat{X}$ & none & 0.172 & 0.686 & 0.004 & 4.03 & 0.102 & 0.408 & 0.457 \\
& & optimal & 0.172 & 0.686 & 0.004 & 1.05 & 0.389 & 0.719 & 0.364 \\
$\delta 3$. & smooth $\delta$ & none & 0.175 & 0.701 & 0.048 & 3.72 & 0.112 & 0.408 & 0.454 \\
& & optimal & 0.174 & 0.697 & 0.027 & 1.03 & 0.404 & 0.713 & 0.358 \\
\hline
\end{tabular}

Table 5-Time Paths of Optimal Child Taxes with Non-Zero Damages

\begin{tabular}{llcccccc}
\multicolumn{1}{c}{ Case } & $\{\tau / y\}_{0}^{*}$ & $\{\tau / y\}_{1}^{*}$ & $\{\tau / y\}_{2}^{*}$ & $\{\tau / y\}_{3}^{*}$ & $\{\tau / y\}_{4}^{*}$ & $\{\tau / y\}_{s s}^{*}$ \\
\hline$\delta 1$. & cap $\hat{E}$ & 0.109 & 0.143 & 0.165 & 0.181 & 0.191 & 0.209 \\
$\delta 2$. & cap $\hat{X}$ & 0.186 & 0.208 & 0.214 & 0.212 & 0.211 & 0.210 \\
$\delta 3$. & smooth $\delta$ & 0.184 & 0.206 & 0.213 & 0.212 & 0.210 & 0.207 \\
\hline
\end{tabular}

we must assume $e^{+}=0.464$, which implies uncapped emissions $E_{0}^{+}=0.386 .{ }^{39}$ The discovery that an emissions freeze is needed thus means $\hat{E}=0.386$.

Case $\delta 1$ (labeled $\delta$ for damage) in tables 4-5 show results for this scenario. Steady state values and the path of tax rates both are quite similar to the base case (case 1 in tables 1-2). This suggests S3 is a reasonable simplification. ${ }^{40}$ Intuitively, when a parent spends almost 25 percent of income on a child $(\psi+\chi=0.248)$, damages of $\delta_{s s}=0.012$ are too small to change fertility significantly.

Second, we drop S2 and assume instead that there is a critical value $\hat{X}$ for greenhouse gas stocks such that (22) applies for $X_{t} \leq \hat{X}$ and $\delta\left(X_{t}\right)=1$ for $X_{t}>\hat{X}$. (S1 still applies, now with $\mathcal{X}=\left\{X_{t}>\hat{X}\right\}$.) For illustration, we set $\hat{X}=\gamma X_{0}+E_{0}^{+}=\tilde{n}^{+} X_{0}=0.686$. This yields a scenario comparable to case $\delta 1$ in the sense that uncapped emissions at $t=0$ would place the economy on the verge of disaster.

Replacing $\hat{E}$ by $\hat{X}$ alters the dynamics between $t=0$ and the steady state. The

\footnotetext{
${ }^{39}$ This follows from $\tilde{n}^{+}=1.716, \gamma=0.75$, and $\tilde{\psi} \tilde{n}^{+}=0.17$.

${ }^{40}$ Assumption $\mathrm{S} 3$ is important to streamline the exposition. Without $\mathrm{S} 3$, the analysis in the previous sections would require a multi-dimensional state space $(\tilde{N}, X)$ even when damages are small.
} 
steady state ratio $E_{s s} / X_{s s}=1-\gamma$ is much less the initial ratio $E_{0}^{+} / X_{0}=\tilde{n}^{+}-\gamma$, because greenhouse gas stocks catch up when emissions stop growing. When emissions are frozen at $t=0$, greenhouse gas stocks will grow to $X_{s s}=\hat{E} /(1-\gamma)=\left(\tilde{n}^{+}-\right.$ $\gamma) X_{0} /(1-\gamma)$; but when stocks are frozen at $X_{s s}=\hat{X}$, emissions must decline to $E_{s s}=$ $(1-\gamma) \hat{X}=0.172$.

Case $\delta 2$ in tables 4-5 show results for $\hat{X}=0.686$. Steady-state population in case $\delta 2$ is less than half of population in case $\delta 1$, roughly in proportion to reduced emissions (56 percent cut from 0.386 to 0.172 ). The per-capita variables in case $\delta 2$ are, however, similar in case $\delta 1$. In table 5 , tax rates for case $\delta 2$ are higher than in $\delta 1$. The intuition is as in the main model: a cut in emissions requires high tax rates during the transition (see row 2 versus row 1 in table 2). In summary, results with cap $\hat{X}$ are similar to results with cap $\hat{E}$, provided one adjusts for differences in steady-state emissions. A substantive insight is that freezing greenhouse gas stocks implies cutting emissions and hence higher child taxes at $t=0$ than in the baseline calibration.

Third, we modify S1 by assuming a smooth damage function that follows (22) for $X_{t} \leq \hat{X}$ and ends at $\delta(\hat{X}+\Delta \hat{X})=1$ for some $\Delta \hat{X}>0$. With smooth damages, determining optimal emissions is is no longer a simple problem, and it is beyond the scope of this paper to survey the range of possible specifications. We present only one scenario for illustration, setting $\hat{X}=0.686, \Delta \hat{X}=\hat{X} / 10$, and assuming a quadratic damage function for $X_{t}>\hat{X}$. Then (22) applies for greenhouse gas levels up to 0.686 , and marginal damages increase linearly on the interval $[\hat{X}, \hat{X}+\Delta \hat{X}]=[0.686,0.762]$. Greenhouse gas stocks are again bounded (now at 0.762), but optimal emissions, optimal greenhouse gas dynamics, and optimal "acceptable" damages are endogenous. Case $\delta 3$ in tables 4-5 shows that steady-state values and tax rates are similar to case $\delta 2$, and damages are higher (due to $X_{s s}>\hat{X}$ ). A notable difference is that steady state emissions take slightly different values with and without child taxes. Intuitively, restricting emissions is more costly in terms of output when fertility is untaxed, so optimal emissions without child taxes are higher $(0.175$ versus 0.174$) .{ }^{41}$ In summary, results with smooth damage

\footnotetext{
${ }^{41}$ Key features of case $\delta 3$ are that greenhouse gas stocks are bounded and that marginal damages are high enough to
} 
function are more complicated than results with catastrophe at $\hat{X}$, but not necessarily much different with regard to population and child taxes.

\section{Concluding Thoughts}

In thinking about population policy, it is useful to know the magnitude of the induced population externality as this equals the tax/subsidy policy that is optimal for currently living individuals. We consider a specific case that suggests population externalities induced by underlying externalities can be large: we take as a maintained hypothesis that global warming is a serious problem and a cap is imposed, and that the problem does not go away because of fortuitous shifts in productivity or a permanent decline in fertility to less than replacement. The induced population externality is generally large.

To speculate beyond this paper, many current tax, welfare, and school policies subsidize children, so it is likely that net policy today is pronatalist. Positive population externalities from pay-as-you-go policies and from scale/spillover effects in human capital formation may partly explain this. To evaluate this more fully and also to understand economic pressures on population policy generally, it may be useful to measure the contributions of different underlying externalities to the aggregate induced population externality. If emissions restrictions are imposed, however, then the analysis here suggests that, all else equal, there may be pressure to make population policy less pronatalist.

\section{REFERENCES}

Alvarez, Fernando. 1999. "Social Mobility: The Barro-Becker Children Meet the Laitner-Loury Dynasties," Review of Economic Dynamics, 2 (1), 65-103.

Bansal, Ravi and Amir Yaron. 2004. "Risks for the Long Run: A Potential Resolution of Asset Pricing Puzzles," Journal of Finance, 59 (4), 1481-1509.

keep equilibrium damages low. If $\Delta \hat{X}$ were higher, marginal damages would be lower and government would optimally accept greater damages. To calibrate such damages, a more elaborate model of the enviroment would be needed.

We thank an anonymous referee for noting that optimal emission generally depend on the availability of child taxes; case $\delta 3$ serves to illustrate this point. 
Barro, Robert. 2013. "Environmental Protection, Rare Disasters, and Discount Rates," NBER working paper w19258. and Gary Becker. 1988. "A Reformulation of the Economic Theory of Fertility," Quarterly Journal of Economics, 103 (1), 1-25.

1989. "Fertility Choice in a Model of Economic Growth," Econometrica, 57 (2), 481-501.

Becker, Gary. 1960. "An Economic Analysis of Fertility," in Demographic and Economic Change in Developed Countries, Princeton: Princeton Univ. Press.

Caldwell, John. 1980. "Mass Education as a Determinant of the Timing of Fertility Decline," Population and Develoment Review, 6, 225-55.

- 2007. Theory of Fertility Decline, New York: Academic Press, 1982.

Golosov, Mikhail, Larry Jones, and Michèle Tertilt. 2007. "Efficiency with Endogenous Population Growth,” Econometrica, 75 (4), 1039-71.

Golosov, Mikhail, John Hassler, Per Krusell, and Aleh Tsyvinski. 2011. “Optimal Taxes on Fossil Fuel in General Equilibrium,” NBER working paper 17348.

Harford, Jon. 1997. "Stock Pollution, Child-Bearing Externalities, and the Social Discount Rate," Journal of Environmental Economics and Management, 33 (1), 94-105. . 1998. “The Ultimate Externality," American Economic Review, 88 (1), 26065. 2000. "Methods of Pricing Common Property Use and Some Implications for Optimal Child-Bearing and the Social Discount Rate," Resource and Energy Economics, 22 (2), 103-24.

IPCC (Intergovernmental Panel on Climate Change). 2008. Fourth Assessment Report, Climate Change 2007: Synthesis Report, Geneva.

Jones, Charles. 1999. "Growth: With or Without Scale Effects?," American Economic Review, 89 (2), 139-44.

Jones, Larry, and Alice Schoonbroodt. 2010. "Complements versus Substitutes and Trends in Fertility Choice in Dynastic Models," International Economic Review, 51(3), 
671-99.

Jones, Larry, Alice Schoonbroodt, and Michèle Tertilt. 2008. "Fertility Theories: Can They Explain the Negative Fertility-Income Relationship," NBER working paper 14266.

Kelly, David and Charles Kolstad. 2001. "Malthus and Climate Change: Betting on Stable Population," Journal of Environmental Economics and Management, 41 (2), 13561.

Kremer, Michael. 1993. "Population Growth and Technological Change: One Million B.C. to 1990," Quarterly Journal of Economics, 108 (3), 681-716.

Nordhaus, William and Joseph Boyer. 2000. Warming the World: the Economics of the Greenhouse Effect, Cambridge, MA: MIT Press. (1999 version available at http://www.econ.yale.edu/ nordhaus/homepage/web table of contents 102599.htm.)

Ogaki, Masao and Carmen Reinhart. 1998. "Measuring Intertemporal Substitution: The Role of Durable Goods," Journal of Political Economy, 106 (5), 1078-98.

Pindyck, Robert. 2013. “Climate Change Policy: What Do the Models Tell Us?”, NBER working paper w19244.

Schoonbroodt, Alice and Michèle Tertilt. 2010. "Property Rights and Efficiency in OLG Models with Endogenous Fertility," working paper.

Stern, Nicholas. 2007. Economics of Climate Change: The Stern Review, Cambridge: Cambridge University Press.

United Nations. 2010. World Population Prospects, the 2010 Revision (Figure 1), online at http://esa.un.org/unpd/wpp/Analytical-Figures/htm/fig_1.htm.

World Resources Institute. 2008. Climate Analysis Indicators Tool (CAIT), version 3.0., Washington, DC: World Resources Institute, http://cait.wri.org. 